\title{
Maximal Regularity for Non-autonomous Equations with Measurable Dependence on Time
}

\author{
Chiara Gallarati ${ }^{1} \cdot$ Mark $_{\text {Veraar }}{ }^{1}$
}

Received: 18 March 2016 / Accepted: 9 September 2016 / Published online: 22 September 2016

(C) The Author(s) 2016. This article is published with open access at Springerlink.com

\begin{abstract}
In this paper we study maximal $L^{p}$-regularity for evolution equations with timedependent operators $A$. We merely assume a measurable dependence on time. In the first part of the paper we present a new sufficient condition for the $L^{p}$-boundedness of a class of vector-valued singular integrals which does not rely on Hörmander conditions in the time variable. This is then used to develop an abstract operator-theoretic approach to maximal regularity. The results are applied to the case of $m$-th order elliptic operators $A$ with time and space-dependent coefficients. Here the highest order coefficients are assumed to be measurable in time and continuous in the space variables. This results in an $L^{p}\left(L^{q}\right)$-theory for such equations for $p, q \in(1, \infty)$. In the final section we extend a well-posedness result for quasilinear equations to the time-dependent setting. Here we give an example of a nonlinear parabolic PDE to which the result can be applied.
\end{abstract}

Keywords Singular integrals $\cdot$ Maximal $L^{p}$-regularity $\cdot$ Evolution equations $\cdot$ Functional calculus $\cdot$ Elliptic operators $\cdot A_{p}$-weights $\cdot \mathcal{R}$-boundedness $\cdot$ Extrapolation $\cdot$ Quasi-linear PDE

Mathematics Subject Classification (2010) Primary: 42B20 • 42B37; Secondary: $34 \mathrm{G} 10 \cdot 35 \mathrm{~B} 65 \cdot 42 \mathrm{~B} 15 \cdot 47 \mathrm{D} 06 \cdot 35 \mathrm{~K} 90 \cdot 34 \mathrm{G} 20 \cdot 35 \mathrm{~K} 55$

The first author is supported by Vrije Competitie subsidy 613.001.206 and the second author by the Vidi subsidy 639.032.427 of the Netherlands Organisation for Scientific Research (NWO)

Mark Veraar

M.C.Veraar@tudelft.nl

Chiara Gallarati

C.Gallarati@tudelft.nl

1 Delft Institute of Applied Mathematics, Delft University of Technology, P.O. Box 5031, 2600 GA Delft, The Netherlands 


\section{Introduction}

In this paper we study maximal $L^{p}$-regularity of the Cauchy problem:

$$
\begin{aligned}
u^{\prime}(t)+A(t) u(t) & =f(t), t \in(0, T) \\
u(0) & =x .
\end{aligned}
$$

Here $(A(t))_{t \in(0, T)}$ is a family of closed operators on a Banach space $X_{0}$. We assume the operators have a constant domain $D(A(t))=X_{1}$ for $t \in[0, T]$.

In recent years there has been much interest in maximal regularity techniques and their application to nonlinear PDEs. Maximal regularity can often be used to obtain a priori estimates which give global existence results. For example, using maximal regularity it is possible to solve quasi-linear and fully nonlinear PDEs by elegant linearization techniques combined with the contraction mapping principle $[4,6,14,16,67,81]$. This has found numerous applications in problems from mathematical physics (e.g. fluid dynamics, reaction-diffusion equations, material science, etc. see e.g. $[1,14,21,35,44,63,70,71,79$, $81,84,85,95])$. For maximal Hölder-regularity we refer the reader to [2, 67] and references therein. In this paper we focus on maximal $L^{p}$-regularity as this usually requires the least regularity of the data in PDEs.

An important step in the theory of maximal $L^{p}$-regularity was the discovery of an operator-theoretic characterization in terms of $\mathcal{R}$-boundedness properties of the differential operator $A$ due to Weis (see [91, 92]). This characterization was proved for the class of Banach spaces with the UMD property. About the same time Kalton and Lancien discovered that not every sectorial operator $A$ on $X=L^{q}$ of angle $<\pi / 2$ has maximal $L^{p}$-regularity (see [53, 54] and [29]), but their example is not a differential operator.

In the case $t \mapsto A(t)$ is (piecewise) continuous, one can study maximal $L^{p}$-regularity using perturbation arguments (see [5, 7, 82]). In particular, in [82], it was shown that maximal $L^{p}$-regularity of Eq. 1.1 is equivalent to the maximal $L^{p}$-regularity for each operator $A\left(t_{0}\right)$ for $t_{0} \in[0, T]$ fixed. This, combined with the characterization of [92] yields a very precise condition for maximal $L^{p}$-regularity. The case where the domains $D(A(t))$ vary in time will not be considered in this paper. In that setting maximal $L^{p}$-regularity results can be obtained under certain Hölder regularity assumptions in the time variable (see [80] and references therein).

In many real-life models, the differential operator $A$ has time-dependent coefficients, and the dependence on time can be rather rough (e.g. the coefficient could be a stochastic process). If this is the case, the operator-theoretic characterization of maximal regularity just mentioned does not apply or leads to unwanted restrictions. In the present paper we develop a functional analytic approach to maximal $L^{p}$-regularity in the case $t \mapsto A(t)$ is only measurable (see Theorems 1.1 and 4.9 below). Our approach is based on the $L^{p}$ boundedness of a new class of vector-valued singular integrals of non-convolution type (see Theorem 3.4). It is important to note that we do not assume any Hörmander conditions on the kernel in the time variable. For discussion and references on (vector-valued) singular integrals we refer the reader to Section 3.

When the time-dependence is just measurable, an operator-theoretic condition for maximal $L^{p}$-regularity is known only in the Hilbert space setting for $p=2$ (see $[65,66]$ and [88, Section 5.5]). The assumption here is that $A$ arises from a coercive form $a(t, \cdot, \cdot)$ : $V \times V \rightarrow \mathbb{C}$ and $V \hookrightarrow X_{0} \hookrightarrow V^{\prime}$. Unfortunately, this only yields a theory of maximal $L^{2}$ regularity on $V^{\prime}$ in general (see [30] for a counterexample). In many situations one would like to have maximal $L^{p}$-regularity on $X_{0}$ and also for any $p \in(1, \infty)$. Results of this type 
have been obtained in $[8,23,24,41]$ using regularity conditions on the form in the time variable.

Most results will be presented in the setting of weighted $L^{p}$-spaces. For instance Theorems 1.2 and 5.4 we will present a weighted $L^{p}\left(L^{q}\right)$-maximal regularity result in the case $A$ is a $2 m$-th order elliptic operator, assuming only measurability in the time variable and continuity in the space variable. Weighted results can be important for several reasons. Maximal $L^{p}$-regularity with a power weight $t^{\alpha}$ in time (e.g. see $[59,71]$ ) allows one to consider rather rough initial values. It can also be used to prove compactness properties which in turn can be used to obtain global existence of solutions. Another advantage of using weights comes from a harmonic analytic point of view. The theory of Rubio de Francia (see [18] and references therein) enables one to extrapolate from weighted $L^{p}$-estimates for a single $p \in(1, \infty)$, to any $p \in(1, \infty)$. In Section $5 A_{p}$-weights in space will be used to check $\mathcal{R}$ boundedness of certain integral operators. We refer to Theorem 2.6 and Step 1 of the proof of Theorem 5.4 for details. Weights in time will be used for extrapolation arguments more directly. For instance in step 4 of the proof of Theorem 5.4 and also the proof of Theorem 1.1 at the end of Section 4.4.

In the special case $X_{0}$ is a Hilbert space, our main result Theorem 4.9 implies the following result.

Theorem 1.1 Let $X_{0}$ be a Hilbert space. Assume $A:(0, \tau) \rightarrow \mathscr{L}\left(X_{1}, X_{0}\right)$ is such that for all $x \in X_{1}, t \mapsto A(t) x$ is measurable and

$$
c_{1}\|x\|_{X_{1}} \leq\|x\|_{X_{0}}+\|A(t) x\|_{X_{0}} \leq c_{2}\|x\|_{X_{1}}, \quad t \in(0, \tau), x \in X_{1} .
$$

Assume there is an operator $A_{0}$ on $X_{0}$ with $D\left(A_{0}\right)=X_{1}$ which generates a contractive analytic semigroup $\left(e^{-z A_{0}}\right)_{z \in \Sigma_{\theta}}$ which is such that $\left(A(t)-A_{0}\right)_{t \in(0, \tau)}$ generates an evolution system $(T(t, s))_{0 \leq s \leq t \leq \tau}$ on $X_{0}$ which commutes with $\left(e^{-r A_{0}}\right)_{r \geq 0}$.

$$
e^{-r A_{0}} T(t, s)=T(t, s) e^{-r A_{0}}, \quad 0 \leq s \leq t \leq \tau, \quad r \geq 0 .
$$

Then A has maximal $L^{p}$-regularity for every $p \in(1, \infty)$, i.e. for every $f \in L^{p}\left(0, \tau ; X_{0}\right)$ and $x \in\left(X_{0}, X_{1}\right)_{1-\frac{1}{p}, p}$ there exists a unique strong solution $u \in L^{p}\left(0, \tau ; X_{1}\right) \cap$ $W^{1, p}\left(0, \tau ; X_{0}\right) \cap C\left([0, \tau] ;\left(X_{0}, X_{1}\right)_{1-\frac{1}{p}, p}\right)$ of Eq. 1.1 and there is a constant $C$ independent of $f$ and $x$ such that

$$
\begin{aligned}
\|u\|_{L^{p}\left(0, \tau ; X_{1}\right)}+\|u\|_{W^{1, p}\left(0, \tau ; X_{0}\right)}+ & \|u\|_{C\left([0, \tau] ;\left(X_{0}, X_{1}\right)_{1-\frac{1}{p}, p}\right)} \\
& \leq C\|f\|_{L^{p}\left(0, \tau ; X_{0}\right)}+C\|x\|_{\left(X_{0}, X_{1}\right)_{1-\frac{1}{p}, p}} .
\end{aligned}
$$

The condition on $A(t)-A_{0}$ can be seen as an abstract ellipticity condition. The assumption that the operators are commuting for instance holds if $A(t)$ and $A_{0}$ are differential operators with coefficients independent of the space variable on $\mathbb{R}^{d}$. We will show that the space dependence can be put in later on by perturbation arguments.

In Section 4.4 we will derive this result from Theorem 4.9 where the case of more general Banach spaces $X_{0}$ and weighted $L^{p}$-spaces is considered. Instead of assuming that $A_{0}$ generates an analytic contraction semigroup one could also assume that $A_{0}$ has a bounded $H^{\infty}$-calculus of angle $<\pi / 2$. 
As an application of our main result we prove maximal $L^{p}$-regularity for the following class of parabolic PDEs:

$$
\begin{aligned}
u^{\prime}(t, x)+A(t) u(t, x) & =f(t, x), t \in(0, T), x \in \mathbb{R}^{d}, \\
u(0, x) & =u_{0}(x), \quad x \in \mathbb{R}^{d} .
\end{aligned}
$$

Here

$$
A(t) u(t, x)=\sum_{|\alpha| \leq m} a_{\alpha}(t, x) D^{\alpha} u(t, x) .
$$

For such concrete equations with coefficients which depend on time in a measurable way, maximal $L^{p}$-regularity results can be derived using PDE techniques. Our results enable us to give an alternative approach to several of these problems. Moreover, we are the first to obtain a full $L^{p}\left(0, T ; L^{q}\left(\mathbb{R}^{d}\right)\right)$-theory, whereas previous papers usually only give results for $p=q$ or $q \leq p$ (see Remark 5.7 for discussion).

In the next result we will use condition (C) on $A$ which will be introduced in Section 5. It basically says that $A$ is uniformly elliptic and the highest order coefficients are continuous in space, but only measurable in time.

Theorem 1.2 Let $T \in(0, \infty)$. Assume condition $(C)$ on the family of operators $(A(t))_{t \in(0, T)}$ given by Eq. 1.3. Let $p, q \in(1, \infty)$. Then the operator $A$ has maximal $L^{p}$-regularity on $(0, T)$, i.e. for every $f \in L^{p}\left(0, T ; L^{q}\left(\mathbb{R}^{d}\right)\right)$ and $u_{0} \in B_{q, p}^{s}\left(\mathbb{R}^{d}\right)$ with $s=m\left(1-\frac{1}{p}\right)$, there exists a unique

$$
u \in W^{1, p}\left(0, T ; L^{q}\left(\mathbb{R}^{d}\right)\right) \cap L^{p}\left(0, T ; W^{m, q}\left(\mathbb{R}^{d}\right)\right) \cap C\left([0, T] ; B_{q, p}^{s}\left(\mathbb{R}^{d}\right)\right)
$$

such that Eq. 1.2 holds a.e. and there is $a C>0$ independent of $u_{0}$ and $f$ such that

$$
\begin{aligned}
\|u\|_{L^{p}\left(0, T ; W^{m, q}\left(\mathbb{R}^{d}\right)\right)}+ & \|u\|_{W^{1, p}\left(0, T ; L^{q}\left(\mathbb{R}^{d}\right)\right)}+\|u\|_{C\left([0, T] ; B_{q, p}^{s}\left(\mathbb{R}^{d}\right)\right)} \\
& \leq C\left(\|f\|_{L^{p}\left(\mathbb{R} ; L^{q}\left(\mathbb{R}^{d}\right)\right)}+\left\|u_{0}\right\|_{B_{q, p}^{s}\left(\mathbb{R}^{d}\right)}\right) .
\end{aligned}
$$

The conditions on $f$ and $u_{0}$ are also necessary in the above result. Here $B_{q, p}^{s}\left(\mathbb{R}^{d}\right)$ denotes the usual Besov space (see [90] for details). The proof of Theorem 1.2 is given at the end of Section 5. It will be derived from Theorem 5.4 which is a maximal regularity result with weights in time and space. One can also consider systems instead of Eq. 1.2. The results in this case are more complicated and will be presented in [33].

Overview In Section 2 we discuss preliminaries on weights, $\mathcal{R}$-boundedness and functional calculus. In Section 3 we prove the $L^{p}$-boundedness of a new class of singular integrals. The main result on maximal $L^{p}$-regularity is presented in Section 4 . In Section 5 we show how to use our new approach to derive maximal $L^{p}$-regularity for Eq. 1.2. Finally in Section 6 we extend the result of [14] and [81] on quasi-linear equations to the time-dependent setting.

Notation Throughout this paper we will write $\mathscr{L}(X, Y)$ for the space of all bounded linear operators mapping $X$ into $Y$. In the estimates below, $C$ can denote a constant which varies from line to line. We set $\mathbb{N}=\{1,2,3, \cdots\}$ and $\mathbb{N}_{0}=\mathbb{N} \cup\{0\}$. 


\section{Preliminaries}

\section{1 $A_{p}$-Weights}

Details on $A_{p}$-weights can be found in [38, Chapter 9] and [87, Chapter V].

A weight is a locally integrable function on $\mathbb{R}^{d}$ with $w(x) \in(0, \infty)$ for a.e. $x \in \mathbb{R}^{d}$. For a Banach space $X$ and $p \in[1, \infty], L^{p}\left(\mathbb{R}^{d}, w ; X\right)$ is the space of all strongly measurable functions $f: \mathbb{R}^{d} \rightarrow X$ such that

$$
\|f\|_{L^{p}\left(\mathbb{R}^{d}, w ; X\right)}=\left(\int_{\mathbb{R}^{d}}\|f(x)\|^{p} w(x) d x\right)^{\frac{1}{p}}<\infty \text { if } p \in[1, \infty),
$$

and $\|f\|_{L^{\infty}\left(\mathbb{R}^{d}, w ; X\right)}=$ ess. $\sup _{x \in \mathbb{R}^{d}}\|f(x)\|$.

For $p \in(1, \infty)$ a weight $w$ is said to be an $A_{p}$-weight if

$$
A_{p}=\sup _{Q} f_{Q} w(x) d x\left(f_{Q} w(x)^{-\frac{1}{p-1}} d x\right)^{p-1}<\infty .
$$

Here the supremum is taken over all cubes $Q \subseteq \mathbb{R}^{d}$ with axes parallel to the coordinate axes and $f_{Q}=\frac{1}{|Q|} \int_{Q}$. The extended real number $[w]_{A_{p}}$ is called the $A_{p}$-constant. The Hardy-Littlewood maximal operator is defined as

$$
M(f)(x)=\sup _{Q \ni x} f_{Q}|f(y)| d y, \quad f \in L^{p}\left(\mathbb{R}^{d}, w\right)
$$

with $Q \subseteq \mathbb{R}^{d}$ cubes as before. Recall that $w \in A_{p}$ if and only if the Hardy-Littlewood maximal operator $M$ is bounded on $L^{p}\left(\mathbb{R}^{d}, w\right)$.

The following simple extension of the extrapolation result from [18, Theorem 3.9] will be needed.

Theorem 2.1 (Extrapolation) For every $\lambda \geq 0$, let $f_{\lambda}, g_{\lambda}: \mathbb{R}^{d} \rightarrow \mathbb{R}_{+}$be a pair of nonnegative, measurable functions and suppose that for some $p_{0} \in(1, \infty)$ there exist increasing functions $\alpha_{p_{0}}, \beta_{p_{0}}$ on $\mathbb{R}_{+}$such that for all $w_{0} \in A_{p_{0}}$ and all $\lambda \geq \beta_{p_{0}}\left(\left[w_{0}\right]_{A_{p_{0}}}\right)$,

$$
\left\|f_{\lambda}\right\|_{L^{p_{0}\left(\mathbb{R}^{d}, w_{0}\right)}} \leq \alpha_{p_{0}}\left(\left[w_{0}\right]_{A_{p_{0}}}\right)\left\|g_{\lambda}\right\|_{L^{p_{0}\left(\mathbb{R}^{d}, w_{0}\right)}} .
$$

Then for all $p \in(1, \infty)$ there is a constant $c_{p, d} \geq 1$ such that for all $w \in A_{p}$, and all $\lambda \geq \beta_{p_{0}}\left(\phi\left([w]_{A_{p}}\right)\right)$

$$
\left\|f_{\lambda}\right\|_{L^{p}\left(\mathbb{R}^{d}, w\right)} \leq 4 \alpha_{p_{0}}\left(\phi\left([w]_{A_{p}}\right)\right)\left\|g_{\lambda}\right\|_{L^{p}\left(\mathbb{R}^{d}, w\right)},
$$

where $\phi(x)=c_{p, d} x^{\frac{p_{0}-1}{p-1}+1}$.

Note that [18, Theorem 3.9] corresponds to the case that $f_{\lambda}$ and $g_{\lambda}$ are constant in $\lambda$. To obtain the above extension one can check that in the proof [18, Theorem 3.9] for given $p$ and $w \in A_{p}$, the $A_{p_{0}}$-weight $w_{0}$ which is constructed satisfies $\left[w_{0}\right]_{A_{p_{0}}} \leq \phi\left([w]_{A_{p}}\right)$. This clarifies the restriction on the $\lambda$ 's.

Below estimates of the form (2.1) with increasing function $\alpha_{p_{0}}$ will appear frequently. In this situation we say there is an $A_{p_{0}}$-consistent constant $C$ such that

$$
\|f\|_{L^{p_{0}\left(\mathbb{R}^{d}, w_{0}\right)}} \leq C\|g\|_{L^{p_{0}\left(\mathbb{R}^{d}, w_{0}\right)}} .
$$

Note that the $L^{p}$-estimate obtained in Theorem 2.1 is again $A_{p}$-consistent for all $p \in$ $(1, \infty)$. 
The following simple observation will be applied frequently. For a bounded Borel set $A \subset \mathbb{R}^{d}$ and for every $f \in L^{p}\left(\mathbb{R}^{d}, w ; X\right)$ one has $\mathbf{1}_{A} f \in L^{1}\left(\mathbb{R}^{d} ; X\right)$ and by Hölder's inequality

$$
\left\|\mathbf{1}_{A} f\right\|_{L^{1}\left(\mathbb{R}^{d} ; X\right)} \leq C_{w, A}\|f\|_{L^{p}\left(\mathbb{R}^{d}, w ; X\right)} .
$$

A linear subspace $Y \subseteq X^{*}$ is said to be norming for $X$ if for all $x \in X,\|x\|=$ $\sup \left\{\left|\left\langle x, x^{*}\right\rangle\right|: x^{*} \in Y,\left\|x^{*}\right\| \leq 1\right\}$. The following simple duality lemma will be needed.

Lemma 2.2 Let $p, p^{\prime} \in[1, \infty]$ be such that $\frac{1}{p}+\frac{1}{p^{\prime}}=1$. Let $v$ be a weight and let $v^{\prime}=v^{-\frac{1}{p-1}}$. Let $Y \subseteq X^{*}$ be a subspace which is norming for $X$. Then setting

$$
\langle f, g\rangle=\int_{\mathbb{R}}\langle f(t), g(t)\rangle d t, \quad f \in L^{p}(\mathbb{R}, v ; X), g \in L^{p^{\prime}}\left(\mathbb{R}, v^{\prime} ; X^{*}\right),
$$

the space $L^{p^{\prime}}\left(\mathbb{R}, v^{\prime} ; X^{*}\right)$ can be isometrically identified with a closed subspace of $L^{p}(\mathbb{R}, v ; X)^{*}$. Moreover, $L^{p^{\prime}}\left(\mathbb{R}, v^{\prime} ; Y\right)$ is norming for $L^{p}(\mathbb{R}, v ; X)$.

\section{2 $\mathcal{R}$-Boundedness and Integral Operators}

In this section we recall the definition of $\mathcal{R}$-boundedness (see [15, 22, 62] for details).

A sequence of independent random variables $\left(r_{n}\right)_{n \geq 1}$ on a probability space $(\Omega, \mathscr{A}, \mathbb{P})$ is called a Rademacher sequence if $\mathbb{P}\left(r_{n}=1\right)=\mathbb{P}\left(r_{n}=-1\right)=\frac{1}{2}$.

Let $X$ and $Y$ be Banach spaces. A family of operators $\mathscr{T} \subseteq \mathscr{L}(X, Y)$ is said to be $\mathcal{R}$ bounded if there exists a constant $C$ such that for all $N \in \mathbb{N}$, all sequences $\left(T_{n}\right)_{n=1}^{N}$ in $\mathscr{T}$ and $\left(x_{n}\right)_{n=1}^{N}$ in $X$,

$$
\left\|\sum_{n=1}^{N} r_{n} T_{n} x_{n}\right\|_{L^{2}(\Omega ; Y)} \leq C\left\|\sum_{n=1}^{N} r_{n} x_{n}\right\|_{L^{2}(\Omega ; X)}
$$

The least possible constant $C$ is called the $\mathcal{R}$-bound of $\mathscr{T}$ and is denoted by $\mathcal{R}(\mathscr{T})$. Recall the Kahane-Khintchine inequalities (see [25, 11.1]): for every $p, q \in(0, \infty)$, there exists a $\kappa_{p, q}>0$ such that

$$
\left\|\sum_{n=1}^{N} r_{n} x_{n}\right\|_{L^{p}(\Omega ; X)} \leq \kappa_{p, q}\left\|\sum_{n=1}^{N} r_{n} x_{n}\right\|_{L^{q}(\Omega ; X)} .
$$

Therefore, the $L^{2}(\Omega ; X)$-norms in Eq. 2.2 can be replaced by $L^{p}(\Omega ; X)$, to obtain an equivalent definition up to a constant depending on $p$.

Every $\mathcal{R}$-bounded family of operators is uniformly bounded. A converse holds for Hilbert spaces $X$ and $Y$ : every uniform bounded family of operators is automatically $\mathcal{R}$-bounded.

The $\mathcal{R}$-boundedness of a certain family of integral operators plays a crucial role in this paper. Let $\mathcal{K}$ be the class of kernels $k \in L^{1}(\mathbb{R})$ for which $|k| * f \leq M f$ for all simple functions $f: \mathbb{R} \rightarrow \mathbb{R}_{+}$, where $M$ denotes the Hardy-Littlewood maximal operator. The next example gives an important class of kernels which are in $\mathcal{K}$. 
Example 2.3 Let $k:(0, \infty) \times \mathbb{R} \rightarrow \mathbb{C}$, be such that $|k(u, t)| \leq h\left(\frac{|t|}{u}\right) \frac{1}{u}, u>0$, where $h \in L^{1}\left(\mathbb{R}_{+}\right) \cap C_{b}\left(\mathbb{R}_{+}\right), h$ has a maximum in $x_{0} \in[0, \infty)$ and $h$ is radially decreasing on $\left[x_{0}, \infty\right)$. Then,

$$
\begin{aligned}
\int_{0}^{\infty} \sup _{|t| \geq x}|k(u, t)| d x & \leq \int_{0}^{\infty} \sup _{t \geq x}\left|h\left(\frac{t}{u}\right)\right| \frac{d x}{u}=\int_{0}^{\infty} \sup _{s \geq \frac{x}{u}}|h(s)| \frac{d x}{u}=\int_{0}^{\infty} \sup _{s \geq y}|h(s)| d y \\
& =\int_{0}^{x_{0}} \sup _{s \geq y}|h(s)| d y+\int_{x_{0}}^{\infty}|h(y)| d y=x_{0}\left|h\left(x_{0}\right)\right|+\|h\|_{L^{1}\left(x_{0}, \infty\right)} .
\end{aligned}
$$

Now by [77, Proposition 4.5] we find $\left\{\frac{k(u, \cdot)}{C}: u>0\right\} \subseteq \mathcal{K}$ with $C=x_{0}\left|h\left(x_{0}\right)\right|+$ $\|h\|_{L^{1}\left(x_{0}, \infty\right)}$.

Suppose $T:\left\{(t, s) \in \mathbb{R}^{2}: t \neq s\right\} \rightarrow \mathscr{L}(X)$ is such that for all $x \in X,(t, s) \mapsto T(t, s) x$ is measurable. For $k \in \mathcal{K}$ let

$$
I_{k T} f(t)=\int_{\mathbb{R}} k(t-s) T(t, s) f(s) d s .
$$

Consider the family of integral operators $\mathscr{I}:=\left\{I_{k T}: k \in \mathcal{K}\right\} \subseteq \mathscr{L}\left(L^{p}(\mathbb{R} ; X)\right)$. The $\mathcal{R}$-boundedness of such families $\mathscr{I}$ of operators will play an important role in Section 3.

Proposition 2.4 If $\{T(t, s): s, t \in \mathbb{R}\}$ is uniformly bounded on $X$, then $\mathscr{I}$ is uniformly bounded on $L^{p}(\mathbb{R}, v ; X)$ for every $p \in(1, \infty)$ and $v \in A_{p}$. Moreover, it is also uniform bounded on $L^{1}(\mathbb{R} ; X)$.

Proof For any $p \in(1, \infty)$, note that

$$
\begin{aligned}
\left\|I_{k T} f(t)\right\|_{X} & \leq \int_{\mathbb{R}}|k(t-s)|\|T(t, s) f(s)\|_{X} d s \\
& \leq C \int_{\mathbb{R}}|k(t-s)|\|f(s)\|_{X} d s \leq C M\left(\|f\|_{X}\right)(t) .
\end{aligned}
$$

for a.e. $t \in \mathbb{R}$. Therefore the uniform boundedness of $I_{k T}$ follows from the boundedness of the maximal operator. The case $v \equiv 1$ and $p=1$ follows from Fubini's theorem and the fact that $\|k\|_{L^{1}(\mathbb{R})} \leq 1$ (see [77, Lemma 4.3]).

The $\mathcal{R}$-boundedness of Eq. 2.4 has the following simple extrapolation property:

Proposition 2.5 Let $p_{0} \in(1, \infty)$. Iffor all $v \in A_{p_{0}}, \mathscr{I} \subseteq \mathscr{L}\left(L^{p_{0}}(\mathbb{R}, v ; X)\right)$ is $\mathcal{R}$-bounded by a constant which is $A_{p_{0}}$-consistent, then for every $p \in(1, \infty)$ and $v \in A_{p}, \mathscr{I} \subseteq$ $\mathscr{L}\left(L^{p}(\mathbb{R}, v ; X)\right)$ is $\mathcal{R}$-bounded by a constant which is $A_{p}$-consistent.

Proof The special structure of $\mathscr{I}$ will not be used in this proof. Let $I_{1}, \ldots, I_{N} \in \mathscr{I}$, $f_{1}, \ldots, f_{N} \in L^{p}(\mathbb{R}, v ; X)$ and let

$$
F_{p}(t)=\left\|\sum_{n=1}^{N} r_{n} I_{n} f_{n}(t)\right\|_{L^{p}(\Omega ; X)} \text { and } G_{p}(t)=\left\|\sum_{n=1}^{N} r_{n} f_{n}(t)\right\|_{L^{p}(\Omega ; X)} .
$$

Then the assumption combined with Fubini's theorem yields that for all $v \in A_{p_{0}}$,

$$
\left\|F_{p_{0}}\right\|_{L^{p_{0}(\mathbb{R}, v)}} \leq C\left\|G_{p_{0}}\right\|_{L^{p_{0}(\mathbb{R}, v)}},
$$


where $C$ is a constant which is $A_{p_{0}}$-consistent. Therefore, by Theorem 2.1 we find that for each $p \in(1, \infty)$, there is an $A_{p}$-consistent constant $C^{\prime}$ (depending only on $C$ ) such that

$$
\left\|F_{p_{0}}\right\|_{L^{p}(\mathbb{R}, v)} \leq C^{\prime}\left\|G_{p_{0}}\right\|_{L^{p}(\mathbb{R}, v)} .
$$

Now by Eq. 2.3, $F_{p} \leq \kappa_{p, p_{0}} F_{p_{0}}, G_{p_{0}} \leq \kappa_{p_{0}, p} G_{p}$, and the result follows from Eq. 2.5 and another application of Fubini's theorem.

In [32] the following simple sufficient condition for $\mathcal{R}$-boundedness of such families was obtained in the case $X=L^{q}$.

Theorem 2.6 Let $\mathcal{O} \subseteq \mathbb{R}^{d}$ be open. Let $q_{0} \in(1, \infty)$ and let $\{T(t, s): s, t \in \mathbb{R}\}$ be a family of bounded operators on $L^{q_{0}}(\mathcal{O})$. Assume that for all $A_{q_{0}}$-weights $w$,

$$
\|T(t, s)\|_{\mathscr{L}\left(L^{q_{0}}(\mathcal{O}, w)\right)} \leq C,
$$

where $C$ is $A_{q_{0}}$-consistent and independent of $t, s \in \mathbb{R}$. Then the family of integral operators $\mathscr{I}=\left\{I_{k T}: k \in \mathcal{K}\right\} \subseteq \mathscr{L}\left(L^{p}\left(\mathbb{R}, v ; L^{q}(\mathcal{O}, w)\right)\right)$ as defined in Eq. 2.4 is $\mathcal{R}$-bounded for all $p, q \in(1, \infty)$ and all $v \in A_{p}$ and $w \in A_{q}$. Moreover, in this case the $\mathcal{R}$-bounds $\mathcal{R}(\mathscr{I})$ are $A_{p}$ - and $A_{q}$-consistent.

The proof of this result is based on extrapolation techniques of Rubio de Francia. As for fixed $t, s \in \mathbb{R}, T(t, s)$ on $L^{q}(\mathcal{O})$ is usually defined by a singular integral of convolution type in $\mathbb{R}^{d}$, one can often apply Calderón-Zygmund theory and multiplier theory to verify (2.6). In this case it is usually not more difficult to prove the boundedness for all $A_{q}$-weights, than just $w=1$. The reason for this is that for large classes of operators, boundedness implies weighted boundedness (see [34, Theorem IV.3.9], [38, Theorem 9.4.6] and [45, Corollary $2.10]$ ). Another situation where weights are used to obtain $\mathcal{R}$-boundedness can be found in $[31,43]$.

Example 2.7 For a bounded measurable function $\theta: \mathbb{R}^{2} \rightarrow \mathbb{C}$ let $T(t, s) f=\theta(t, s) f$, $f \in L^{q_{0}}\left(\mathbb{R}^{d}, w\right)$. Then Eq. 2.6 holds and hence Theorem 2.6 implies that $\mathscr{I} \subseteq$ $\mathscr{L}\left(L^{p}\left(\mathbb{R}, v ; L^{q}\left(\mathbb{R}^{d}, w\right)\right)\right)$ is $\mathcal{R}$-bounded for all $p, q \in(1, \infty)$ and all $v \in A_{p}$ and $w \in A_{q}$.

\subsection{Sectorial Operators and $\boldsymbol{H}^{\infty}$-Calculus}

Let $X$ be a Banach space. We briefly recall the definition of the $H^{\infty}$-calculus which was developed by McIntosh and collaborators (see e.g. [3, 9, 17, 68]). We refer to [42, 62] for an extensive treatment of the subject. For $\theta \in(0, \pi)$ we set

$$
\Sigma_{\theta}=\{z \in \mathbb{C} \backslash\{0\}:|\arg (z)|<\theta\},
$$

where $\arg : \mathbb{C} \backslash\{0\} \rightarrow(-\pi, \pi]$. A closed densely defined linear operator $(A, D(A))$ on $X$ is said to be sectorial of type $\sigma \in(0, \pi)$ if it is injective and has dense range, its spectrum is contained in $\overline{\Sigma_{\sigma}}$, and for all $\sigma^{\prime} \in(\sigma, \pi)$ the set

$$
\left\{z(z+A)^{-1}: z \in \mathbb{C} \backslash\{0\},|\arg (z)|>\sigma^{\prime}\right\}
$$

is uniformly bounded by some constant $C_{A}$. The infimum of all $\sigma \in(0, \pi)$ such that $A$ is sectorial of type $\sigma$ is called the sectoriality angle of $A$. If $\sigma<\pi / 2$, then by [67, Proposition 2.1.1], $A$ generates an analytic strongly continuous semigroup $T(z)=e^{-z A}$ for $\arg (z)<$ $\pi / 2-\sigma$ and

$$
\|T(t)\| \leq C_{A} C_{\sigma}, \quad t \geq 0 .
$$


Let $H^{\infty}\left(\Sigma_{\theta}\right)$ denote the Banach space of all bounded analytic functions $f: \Sigma_{\theta} \rightarrow \mathbb{C}$, endowed with the supremum norm. Let $H_{0}^{\infty}\left(\Sigma_{\theta}\right)$ denote the linear subspace of all $f \in$ $H^{\infty}\left(\Sigma_{\theta}\right)$ for which there exists $\varepsilon>0$ and $C \geq 0$ such that

$$
|f(z)| \leq \frac{C|z|^{\varepsilon}}{(1+|z|)^{2 \varepsilon}}, \quad z \in \Sigma_{\theta} .
$$

If $A$ is sectorial of type $\sigma_{0} \in(0, \pi)$, then for all $\sigma \in\left(\sigma_{0}, \pi\right)$ and $f \in H_{0}^{\infty}\left(\Sigma_{\sigma}\right)$ we define the bounded operator $f(A)$ by

$$
f(A)=\frac{1}{2 \pi i} \int_{\partial \Sigma_{\sigma}} f(z)(z+A)^{-1} d z .
$$

A sectorial operator $A$ of type $\sigma_{0} \in(0, \pi)$ is said to have a bounded $H^{\infty}\left(\Sigma_{\sigma}\right)$-calculus) for $\sigma \in\left(\sigma_{0}, \pi\right)$ if there exists a $C \geq 0$ such that

$$
\|f(A)\| \leq C\|f\|_{H^{\infty}\left(\Sigma_{\sigma}\right)}, \quad f \in H_{0}^{\infty}\left(\Sigma_{\sigma}\right) .
$$

If $A$ has a bounded $H^{\infty}\left(\Sigma_{\sigma}\right)$-calculus, then the mapping $f \mapsto f(A)$ extends to a bounded algebra homomorphism from $H^{\infty}\left(\Sigma_{\sigma}\right)$ to $\mathscr{L}(X)$ of norm $\leq C$.

Many differential operators on $L^{q}$-spaces with $q \in(1, \infty)$ are known to have a bounded $H^{\infty}$-calculus (see [22,62] and the survey [93]). The case $A=-\Delta$ on $L^{p}\left(\mathbb{R}^{d}, w\right)$ has a bounded $H^{\infty}$-calculus of arbitrary small angle $\sigma \in(0, \pi)$ for every $w \in A_{p}$ and $p \in(1, \infty)$. This easily follows from the weighted version of Mihlin's multiplier theorem (see [62, Example 10.2] and [34, Theorem IV.3.9]). For instance, it includes all sectorial operators $A$ of angle $<\pi / 2$ for which $e^{-t A}$ is a positive contraction (see [56]).

\section{A Class of Singular Integrals with Operator-Valued Kernel}

Let $X$ be a Banach space. In this section we will study a class of singular integrals of the form

$$
I_{K} f(t)=\int_{\mathbb{R}} K(t, s) f(s) d s, \quad t \in \mathbb{R},
$$

where $K:\{(t, s): t \neq s\} \rightarrow \mathscr{L}(X)$ is an operator-valued kernel. If a kernel $L$ depends on one variable we write $I_{L}=I_{K}$ where $K(t, s)=L(t-s)$.

There is a natural generalization of the theory of singular integrals of convolution type to the vector-valued setting (see [50]). In the case the singular integral is of non-convolution type, the situation is much more complicated. An extensive treatment can be found in [47-49], where $T$ 1-theorems [19] and $T b$-theorems [20] have been obtained in an infinite dimensional setting. Checking the conditions of these theorems can be hard. For instance, from [69] it follows that the typical BMO conditions one needs to check, have a different behavior in infinite dimensions. Our motivation comes from the application to maximal $L^{p}$-regularity of Eq. 1.2. At the moment we do not know whether the $T 1$-theorem and $T b$ theorem can be applied to study maximal $L^{p}$-regularity for the time dependent problems we consider. Below we study a special class of singular integrals with operator-valued kernel for which we prove $L^{p}$-boundedness. The assumptions on $K$ are formulated in such a way that they are suitable for proving maximal $L^{p}$-regularity of Eq. 1.2 later on.

\subsection{Assumptions}

The assumptions in the main result of this section are as follows. 
(H1) Let $X$ be a Banach space and let $p \in[1, \infty)$ and $^{1} v \in A_{p}$.

(H2) The kernel $K$ factorizes as

$$
K(t, s)=\frac{\phi_{0}\left(|t-s| A_{0}\right) T(t, s) \phi_{1}\left(|t-s| A_{1}\right)}{t-s}, \quad(t, s) \in \mathbb{R}^{2}, t \neq s .
$$

Here $A_{0}$ and $A_{1}$ are sectorial operators on $X$ of angle $<\sigma_{0}$ and $<\sigma_{1}$ respectively, and $\phi_{j} \in H_{0}^{\infty}\left(\Sigma_{\sigma_{j}^{\prime}}\right)$ and $\sigma_{j}^{\prime} \in\left(\sigma_{j}, \pi\right)$ for $j=0,1$. Moreover, we assume $T$ : $\{(t, s): t \neq s\} \rightarrow \mathscr{L}(X)$ is uniformly bounded and for all $x \in X,\{(t, s): t \neq$ $s\} \mapsto T(t, s) x$ is strongly measurable.

(H3) Assume $X$ has finite cotype. Assume $A_{j}$ has a bounded $H^{\infty}\left(\Sigma_{\sigma_{j}}\right)$-calculus with $\sigma_{j} \in[0, \pi)$ for $j=0,1$.

(H4) Assume the family of integral operators $\mathscr{I}:=\left\{I_{k T}: k \in \mathcal{K}\right\} \subseteq \mathscr{L}\left(L^{p}(\mathbb{R}, v ; X)\right)$ is $\mathcal{R}$-bounded.

The class of kernels $\mathcal{K}$ is as defined in Section 2.2. Recall from Eq. 3.1 that

$$
I_{k T} f(t)=\int_{\mathbb{R}} k(t-s) T(t, s) f(s) d s .
$$

Since $T$ is uniformly bounded, the operator $I_{k T}$ is bounded on $L^{p}(\mathbb{R}, v ; X)$ by Proposition 2.4.

\section{Remark 3.1}

1. The class of Banach spaces with finite cotype is rather large. It contains all $L^{p}$-spaces, Sobolev, Besov and Hardy spaces as long as the integrability exponents are in the range $[1, \infty)$. The spaces $c_{0}$ and $L^{\infty}$ do not have finite cotype. The cotype of $X$ will be applied in order to have estimates for certain continuous square functions (see Eq. 3.7). Details on type and cotype can be found in [25].

2. In the theory of singular integrals in a vector-valued setting one usually assumes $X$ is a UMD space. Note that every UMD has finite cotype and nontrivial type by the Maurey-Pisier theorem (see [25]).

3. A sufficient condition for the $R$-boundedness condition in the case $X=L^{q}$ can be deduced from Theorem 2.6.

4. In $(\mathrm{H} 2), \phi_{j}\left(|t-s| A_{j}\right)$ could be replaced by $\phi_{j}\left((t-s) A_{j}\right)$ if the $A_{j}$ 's are bisectorial operators. On the other hand, one can also consider $T(t, s) \mathbf{1}_{\{s<t\}}$ and $T(t, s) \mathbf{1}_{\{t<s\}}$ separately. Indeed, the hypothesis $(\mathrm{H} 1)-(\mathrm{H} 4)$ holds for these operators as well whenever they hold for $T(t, s)$.

Example 3.2 Typical examples of functions $\phi_{j}$ which one can take are $\phi_{j}(z)=z^{\alpha} e^{-z}$ for $j=0,1$. If $T(t, s)=I \mathbf{1}_{\{s<t\}}$, then for $A=A_{0}=A_{1}$ one would have

$$
K(t, s)=(t-s)^{2 \alpha-1} A^{2 \alpha} e^{-2(t-s) A} \mathbf{1}_{\{s<t\}} .
$$

This kernel satisfies $\|K(t, s)\| \sim(t-s)^{-1}$ for $t$ close to $s$. If one takes $T(t, s)$ varying in $t$ and $s$ one might view it as a multiplicative perturbation of the above kernel.

\footnotetext{
${ }^{1}$ For the case $p=1$, the convention will be that $v \equiv 1$.
} 
The following simple observation shows that $I_{K}$ as given in Eq. 3.1 can be defined on $L^{p}\left(\mathbb{R} ; D\left(A_{1}\right) \cap R\left(A_{1}\right)\right)$, where $D\left(A_{1}\right)$ denotes the domain of $A_{1}$ and $R\left(A_{1}\right)$ the range of $A_{1}$.

Lemma 3.3 Under the assumptions $(H 1)$ and $(H 2), I_{K}$ is bounded as an operator from $L^{p}\left(\mathbb{R}, v ; D\left(A_{1}\right) \cap R\left(A_{1}\right)\right)$ into $L^{p}(\mathbb{R}, v ; X)$.

Proof As $\phi_{1} \in H_{0}^{\infty}\left(\Sigma_{\sigma_{1}^{\prime}}\right)$ we can find a constant $C$ and $\varepsilon \in(0,1)$ such that $\left|\phi_{1}(z)\right| \leq$ $C|z|^{\varepsilon}|1+z|^{-2 \varepsilon}$. One can check that for all $x \in D\left(A_{1}\right) \cap R\left(A_{1}\right)$,

$$
\left\|\phi_{1}\left(t A_{1}\right) x\right\| \leq C \min \left\{t^{\varepsilon}, t^{-\varepsilon}\right\}\left(\|x\|+\left\|A_{1} x\right\|+\left\|A_{1}^{-1} x\right\|\right), \quad t>0 .
$$

Now since $\phi_{0} \in H_{0}^{\infty}\left(\Sigma_{\sigma_{0}^{\prime}}\right)$ and $\|T(t, s)\|$ is uniformly bounded we obtain

$$
\begin{aligned}
|t-s|\|K(t, s) x\| & \leq\left\|\phi_{0}\left(|t-s| A_{0}\right)\right\|\|T(t, s)\|\left\|\phi_{1}\left(|t-s| A_{1}\right) x\right\| \\
& \leq C \min \left\{|t-s|^{\varepsilon},|t-s|^{-\varepsilon}\right\}\left(\|x\|+\left\|A_{1} x\right\|+\left\|A_{1}^{-1} x\right\|\right) .
\end{aligned}
$$

Therefore, $K:\{(t, s): t \neq s\} \rightarrow \mathscr{L}\left(R\left(A_{1}\right) \cap D\left(A_{1}\right), X\right)$ is essentially nonsingular, and the assertion of the lemma easily follows from [37, Theorem 2.1.10] and the boundedness of the Hardy-Littlewood maximal operator for $p \in(1, \infty)$. The case $p=1$ follows from Young's inequality.

\subsection{Main Result on Singular Integrals}

Theorem 3.4 Assume (H1)-(H4). Then $I_{K}$ defined by Eq. 3.1 extends to a bounded operator on $L^{p}(\mathbb{R}, v ; X)$.

The proof is inspired by the recent solution to the stochastic maximal $L^{p}$-regularity problem given in [76].

Before we turn to the proof, we have some preliminary results and remarks.

Example 3.5 Assume (H2) and (H3). If $T(t, s)$ is as in Example 2.7 then (H4) holds. Therefore, $I_{K}$ is bounded by Theorem 3.4. Surprisingly, we do not need any smoothness of the mapping $(t, s) \mapsto K(t, s)$ in this result. In particular we do not need any regularity conditions for $K(t, s)$ (such as Hörmander's condition) in $(t, s)$.

Recall the following Poisson representation formula (see [76, Lemma 4.1]).

Lemma 3.6 Let $\alpha \in(0, \pi)$ and $\alpha^{\prime} \in(\alpha, \pi]$ be given, let $E$ be a Banach space and let $f: \Sigma_{\alpha^{\prime}} \rightarrow E$ be a bounded analytic function. Then, for all $s>0$ we have

$$
f(s)=\sum_{j \in\{-1,1\}} \frac{j}{2} \int_{0}^{\infty} k_{\alpha}(u, s) f\left(u e^{i j \alpha}\right) d u,
$$

where $k_{\alpha}: \mathbb{R}_{+} \times \mathbb{R}_{+} \rightarrow \mathbb{R}$ is given by

$$
k_{\alpha}(u, t)=\frac{(t / u)^{\frac{\pi}{2 \alpha}}}{(t / u)^{\frac{\pi}{\alpha}}+1} \frac{1}{\alpha u} .
$$

Remark 3.7 In the special case $X=L^{q}(S)$ with $q \in(1, \infty)$, we present some identification of spaces which can be used to simplify the proof below. This might be of use to readers 
who are only interested in $L^{q}$-spaces. First of all one can use the usual adjoint * instead of the moon adjoint \# in the proof below. In this case one can take

$$
\begin{aligned}
\gamma\left(\frac{d u}{u} ; X\right) & =L^{q}\left(S ; L^{2}\left(0, \infty ; \frac{d u}{u}\right)\right), \\
\gamma\left(\frac{d u}{u} ; X\right)^{*} & =L^{q^{\prime}}\left(S ; L^{2}\left(0, \infty ; \frac{d u}{u}\right)\right), \\
\gamma\left(\frac{d u}{u} ; L^{p}(\mathbb{R}, v ; X)\right) & =L^{p}\left(\mathbb{R}, v ; L^{q}\left(S ; L^{2}\left(0, \infty ; \frac{d u}{u}\right)\right)\right) .
\end{aligned}
$$

The $\gamma$-multiplier theorem which is applied below in Eq. 3.6 can be replaced by $[91,4 \mathrm{a}]$ in this case. Finally, the estimates in Eq. 3.7 can be found in [64] in this special case.

Proof of Theorem 3.4 Step 1: By density it suffices to prove $\left\|I_{K} f\right\|_{L^{p}(\mathbb{R}, v ; X)} \leq$ $C\|f\|_{L^{p}(\mathbb{R}, v ; X)}$ with $C$ independent of $f \in L^{p}\left(\mathbb{R}, v ; D\left(A_{1}\right) \cap R\left(A_{1}\right)\right)$. Note that by Lemma $3.3, I_{K}$ is well defined on this subspace.

Step 2: Fix $0<\alpha<\alpha^{\prime} \leq \min \left\{\sigma_{0}^{\prime}-\sigma_{0}, \sigma_{1}^{\prime}-\sigma_{1}\right\}$. First, since $z \rightarrow \phi_{0}\left(z A_{0}\right) T(t, s) \phi_{1}\left(z A_{1}\right)$ is analytic and bounded on $\Sigma_{\alpha^{\prime}}$, by Lemma 3.6, for $x \in D\left(A_{1}\right) \cap R\left(A_{1}\right)$ and $z>0$,

$$
\phi_{0}\left(z A_{0}\right) T(t, s) \phi_{1}\left(z A_{1}\right) x=\sum_{j \in\{-1,1\}} \frac{j}{2} \int_{0}^{\infty} \Phi_{0, j}(u) k_{\alpha}(u, z) T(t, s) \Phi_{1, j}(u) x d u
$$

with $k_{\alpha}(u, t)$ as in Eq. 3.5 and $\Phi_{k, j}(u)=\phi_{k}\left(u e^{i j \alpha} A_{k}\right)$ for $j \in\{-1,1\}$ and $k \in\{0,1\}$. Together with (H2) this yields the following representation of $K(t, s) x$ for $x \in D\left(A_{1}\right) \cap$ $R\left(A_{1}\right)$ :

$$
K(t, s) x=\sum_{j \in\{-1,1\}} \frac{j}{2} \int_{0}^{\infty} \Phi_{0, j}(u) S_{u}(t, s) \Phi_{1, j}(u) x \frac{d u}{u},
$$

where $S_{u}(t, s):=\tilde{k}_{\alpha}(u, t-s) T(t, s)$ with $\tilde{k}_{\alpha}(u, t):=k_{\alpha}(u,|t|) \frac{u}{t}$ and $k_{\alpha}$ is defined as in Eq. 3.5. Moreover, the kernels $\tilde{k}_{\alpha}(u, \cdot)$ satisfy

$$
\left|\tilde{k}_{\alpha}(u, t)\right| \leq \alpha^{-1} h_{\alpha}\left(\frac{t}{u}\right) u^{-1}, \quad u, t>0,
$$

where $h_{\alpha}(x)=\frac{x^{\beta-1}}{x^{2 \beta}+1}$ and $\beta:=\frac{\pi}{2 \alpha}>0$. Extending $k_{\alpha}(u, t)$ as zero for $t<0$, by Example 2.3 we find that $\tilde{k}_{\alpha}(u, \cdot) \in \mathcal{K}$. Indeed, substituting $y=x^{\beta}$, we obtain

$$
\|h\|_{L^{1}(0, \infty)}=\int_{0}^{\infty} \frac{x^{\beta-1}}{x^{2 \beta}+1} d x=\frac{1}{\beta} \int_{0}^{\infty} \frac{1}{y^{2}+1} d y=\alpha .
$$

Therefore, the following representation holds for the singular integral

$$
I_{K} f=\sum_{j \in\{-1,1\}} \frac{j}{2} \int_{0}^{\infty} \Phi_{0, j}(u) I_{S_{u}}\left[\Phi_{1, j}(u) f\right] \frac{d u}{u},
$$

where $f \in L^{p}\left(\mathbb{R}, v ; D\left(A_{1}\right) \cap R\left(A_{1}\right)\right)$.

Step 3: Let $Y_{1}=L^{p}(\mathbb{R}, v ; X)$ and $Y_{2}=L^{p^{\prime}}\left(\mathbb{R}, v^{\prime} ; X^{\#}\right)$, where $X^{\#}=\overline{D\left(A_{0}^{*}\right)} \cap \overline{R\left(A_{0}^{*}\right)}$ is the moondual of $X$ with respect to $A_{0}$ (see [62, Appendix A]) and $v^{\prime}=v^{-\frac{1}{p-1}}$. For $g \in Y_{2}$ write $\langle f, g\rangle_{Y_{1}, Y_{2}}=\int_{\mathbb{R}}\langle f(t), g(t)\rangle d t$. In this way $Y_{2}$ can be identified with an isometric closed subspace of $Y_{1}^{*}$. Note that by [62, Proposition 15.4], $X^{\#}$ is norming for $X$ and hence 
Lemma 2.2 implies that $Y_{2}$ is norming for $Y_{1}$. For fixed $g \in Y_{2}$ it follows from Fubini's theorem and $\gamma$-duality (see [40, Sections 2.3 and 2.6] and [55, Section 5]),

$$
\begin{aligned}
\left|\left\langle I_{K} f, g\right\rangle_{Y_{1}, Y_{2}}\right| & \leq \sum_{j \in\{-1,1\}} \frac{1}{2}\left|\int_{\mathbb{R}} \int_{0}^{\infty}\left\langle\Phi_{0, j}(u) I_{S_{u}}\left[\Phi_{1, j}(u) f\right](t), g(t)\right\rangle \frac{d u}{u} d t\right| \\
& =\sum_{j \in\{-1,1\}} \frac{1}{2}\left|\int_{0}^{\infty}\left\langle I_{S_{u}}\left[\Phi_{1, j}(u) f\right], \Phi_{0, j}(u)^{\#} g\right\rangle \frac{d u}{u}\right| \\
& =\sum_{j \in\{-1,1\}} \frac{1}{2}\left\|I_{S_{u}}\left[\Phi_{1, j}(u) f\right]\right\|_{\gamma\left(\mathbb{R}_{+}, \frac{d u}{u} ; Y_{1}\right)}\left\|\Phi_{0, j}(u)^{\#} g\right\|_{\gamma\left(\mathbb{R}_{+}, \frac{d u}{u} ; Y_{1}\right)^{*} \cdot}
\end{aligned}
$$

Here $\Phi_{0, j}(u)^{\#}:=\phi_{0}\left(u e^{i j \alpha} A_{0}^{\#}\right)$. By (H4) the family $\left\{I_{S_{u}}: u>0\right\}$ is $\mathcal{R}$-bounded by some constant $C_{T}$. Therefore, by the Kalton-Weis $\gamma$-multiplier theorem (see [55, Proposition 4.11] and [74, Theorem 5.2])

$$
\left\|I_{S_{u}}\left[\Phi_{1, j}(u) f\right]\right\|_{\gamma\left(\mathbb{R}_{+}, \frac{d u}{u} ; Y_{1}\right)} \leq C_{T}\left\|\Phi_{1, j}(u) f\right\|_{\gamma\left(\mathbb{R}_{+}, \frac{d u}{u} ; Y_{1}\right)} .
$$

Here we used that $X$ does not contain an isomorphic copy of $c_{0}$ as it has finite cotype (see (H3)). The remaining two square function norms can be estimated by the square function estimates of Kalton and Weis. Indeed, by (H3) and [40, Theorem 4.11] or [55, Section 7] (here we again use the finite cotype of $X$ ) and the $\gamma$-Fubini property (see [74, Theorem 13.6]), we obtain

$$
\begin{aligned}
\left\|\Phi_{1, j}(u) f\right\|_{\gamma_{\left(\mathbb{R}_{+}, \frac{d u}{u} ; Y_{1}\right)}} & \approx\left\|\Phi_{1, j}(u) f\right\|_{L^{p}\left(\mathbb{R}, v ; \gamma\left(\mathbb{R}_{+}, \frac{d u}{u} ; X\right)\right)} \leq C_{A_{1}}\|f\|_{Y_{1}}, \\
\left\|\Phi_{0, j}(u)^{\#} g\right\|_{\gamma\left(\mathbb{R}_{+}, \frac{d u}{u} ; Y_{1}\right)^{*}} & \approx\left\|\Phi_{0, j}(u)^{\#} g\right\|_{L^{p^{\prime}}\left(\mathbb{R}, v^{\prime} ; \gamma\left(\mathbb{R}_{+}, \frac{d u}{u} ; X\right)^{*}\right)} \leq C_{A_{0}}\|g\|_{Y_{2}} .
\end{aligned}
$$

Combining all the estimates yields

$$
\left|\left\langle I_{K} f, g\right\rangle_{Y_{1}, Y_{2}}\right| \leq C_{T} C_{A_{0}} C_{A_{1}}\|f\|_{Y_{1}}\|g\|_{Y_{2}} .
$$

Taking the supremum over all $g \in L^{p^{\prime}}\left(\mathbb{R}, v^{\prime} ; X^{\#}\right)$ with $\|g\|_{Y_{2}} \leq 1$ we find $\left\|I_{K} f\right\|_{Y_{1}} \leq$ $C_{T} C_{A_{0}} C_{A_{1}}\|f\|_{Y_{1}}$. This proves the $L^{p}$-boundedness.

Remark 3.8 One can also apply standard extrapolation techniques to obtain weighted boundedness results for singular integrals from the unweighted case (see $[13,45])$. However, for this one needs Hörmander conditions on the kernel. As our proof gives a result in the more general setting, we can avoid smoothness assumptions on the kernel.

\section{Maximal $L^{p}$-Regularity}

In this section we will apply Theorem 3.4 to obtain maximal $L^{p}$-regularity for the following evolution equation on a Banach space $X_{0}$.

$$
\begin{aligned}
u^{\prime}(t)+A(t) u(t) & =f(t), t \in(0, T) \\
u(0) & =x .
\end{aligned}
$$

As explained in the introduction no abstract $L^{p}$-theory is available for Eq. 4.1 outside the case where $t \mapsto A(t)$ is continuous.

The following assumption will be made throughout this whole section. 
(A) Let $X_{0}$ be a Banach space and assume the Banach space $X_{1}$ embeds densely and continuously in $X_{0}$. Let $p \in[1, \infty)$ and $v \in A_{p}$ with the convention that $v \equiv 1$ if $p=1$. Let $A: \mathbb{R} \rightarrow \mathscr{L}\left(X_{1}, X_{0}\right)$ be such that for all $x \in X_{1}, t \mapsto A(t) x$ is strongly measurable, and there is a constant $C>0$ such that

$$
C^{-1}\|x\|_{X_{1}} \leq\|x\|_{X_{0}}+\|A(t) x\|_{X_{0}} \leq C\|x\|_{X_{1}} .
$$

The above implies that each $A(t)$ is a closed operator on $X_{0}$ with $D(A(t))=X_{1}$. Note that whenever $A$ is given on an interval $I \subseteq \mathbb{R}$, we may always extend it constantly or periodically to all of $\mathbb{R}$.

Before we state the main result we will present some preliminary results on evolution equations with time-dependent $A$.

\subsection{Preliminaries on Evolution Equations}

Evolution equations and evolution families are extensively studied in the literature (see [2, $28,67,78,86,88,89,94])$. We explain some parts which are different in our set-up.

For a strongly measurable function $f:(a, b) \rightarrow X_{0}$ we consider:

$$
\left\{\begin{array}{l}
u^{\prime}(t)+A(t) u(t)=f(t), t \in(a, b) \\
u(a)=x,
\end{array}\right.
$$

where $u(a)=x$ is omitted if $a=-\infty$.

(1) Assume $-\infty<a<b<\infty$. The function $u$ is said to be a strong solution of Eq. 4.2 if $u \in W^{1,1}\left(a, b ; X_{0}\right) \cap L^{1}\left(a, b ; X_{1}\right) \cap C\left([a, b] ; X_{0}\right), u(a)=x$ and Eq. 4.2 holds for almost all $t \in(a, b)$.

(2) Assume $a=-\infty$ and $b<\infty$. The function $u$ is said to be a strong solution of Eq. 4.2 if $u \in W_{\mathrm{loc}}^{1,1}\left(a, b ; X_{0}\right) \cap L_{\mathrm{loc}}^{1}\left(a, b ; X_{1}\right) \cap C\left((a, b] ; X_{0}\right)$ and $\lim _{s \rightarrow a} u(s)=0$ and Eq. 4.2 holds for almost all $t \in(a, b)$.

(3) Assume $b=\infty$. The function $u$ is said to be a strong solution of Eq. 4.2 if for every $T>a$ the restriction to $[a, T]$ or $(a, T]$ yield strong solutions in the sense of (1) and (2) respectively.

Note the following simple embedding result for general $A_{p}$-weights.

Lemma 4.1 Let $p \in[1, \infty)$ and let $v \in A_{p}$, where $v \equiv 1$ if $p=1$. For $-\infty<a<b<\infty$, $W^{1, p}\left((a, b), v ; X_{0}\right) \hookrightarrow C\left([a, b] ; X_{0}\right)$ and

$$
\|u\|_{C\left([a, b] ; X_{0}\right)} \leq C\|u\|_{W^{1, p}\left((a, b), v ; X_{0}\right)} .
$$

Proof Since $L^{p}\left((a, b), v ; X_{0}\right) \hookrightarrow L^{1}\left(a, b ; X_{0}\right)$, and $u(t)-u(s)=\int_{s}^{t} u^{\prime}(r) d r$, the continuity of $u$ is immediate. Moreover,

$$
\|u(t)\| \leq\|u(s)\|+\int_{s}^{t}\left\|u^{\prime}(r)\right\| d r \leq\|u(s)\|+C\left\|u^{\prime}\right\|_{L^{p}\left((a, b), v ; X_{0}\right)} .
$$

Taking $L^{p}((a, b), v)$-norms with respect to the $s$-variable yields the result.

There is a correspondence between the evolution problem (4.2) and evolution families as defined below. 
Definition 4.2 Let $(A(t))_{t \in \mathbb{R}}$ be as in $(A)$. A two parameter family of bounded linear operators $S(t, s), s \leq t$, on a Banach space $X_{0}$ is called an evolution system for $A$ if the following conditions are satisfied:

(i) $S(s, s)=I, S(t, r) S(r, s)=S(t, s)$ for $s \leq r \leq t$;

(ii) $(t, s) \rightarrow S(t, s)$ is strongly continuous for $s \leq t$.

(iii) For all $s \in \mathbb{R}$ and $T \in(s, \infty)$, for all $x \in X_{1}$, the function $u:[s, T] \rightarrow X_{0}$ defined by $u(t)=S(t, s) x$ is in $L^{1}\left(s, T ; X_{1}\right) \cap W^{1,1}\left(s, T ; X_{0}\right)$ and satisfies $u^{\prime}(t)+$ $A(t) S(t, s) x=0$ for almost all $t \in(s, T)$.

(iv) For all $t \in \mathbb{R}$ and $T \in(-\infty, t]$ for all $x \in X_{1}$, the function $u:[T, t] \rightarrow X_{0}$ defined by $u(s)=S(t, s) x$ is in $L^{1}\left(T, t ; X_{1}\right) \cap W^{1,1}\left(T, t ; X_{0}\right)$ and satisfies $u^{\prime}(s)=$ $S(t, s) A(s) x$.

Note that (iii) says that $u$ is a strong solution of Eq. 4.2 with $f=0$.

Example 4.3 If $A(t)=A$ is independent of $t$ and sectorial of angle $<\pi / 2$, then $S(t, s)=$ $e^{-(t-s) A}$ and the two-parameter family of operators reduces to the one-parameter family $e^{-t A}, t \geq 0$, which is the semigroup generated by $-A$.

Example 4.4 Assume $A: \mathbb{R} \rightarrow \mathscr{L}\left(X_{1}, X_{0}\right)$ is strongly measurable and satisfies (A). Define a family of operators $\mathscr{A}$ by

$$
\mathscr{A}=\{A(t): t \in \mathbb{R}\} \cup\left\{\frac{1}{t-s} \int_{s}^{t} A(r) d r: s<t\right\} .
$$

Here we use the strong operator topology to define the integral. Assume there exist $\phi, M$ and $N$ such that all $B \in \mathscr{A}$ are all sectorial of angle $\phi<\pi / 2$ and for all $\lambda \in \Sigma_{\phi}$,

$$
\left\|\lambda(\lambda+B)^{-1}\right\| \leq M \text { and }\|x\|_{X_{1}} \leq N\left(\|x\|_{X_{0}}+\|B x\|_{X_{0}}\right)
$$

Assume for every $B_{1}, B_{2} \in \mathscr{A}$ and $\lambda, \mu \in \Sigma_{\phi}$, the operators $\left(\lambda+B_{1}\right)^{-1}$ and $\left(\mu+B_{2}\right)^{-1}$ commute. Define $S(t, s)=e^{-(t-s) A_{s t}}$, where $A_{s t}=\frac{1}{t-s} \int_{s}^{t} A(r) d r$. Then $S$ is an evolution family for $A$. Here the exponential operator is defined by the usual Cauchy integral (see [67, Chapter 2]). Usually, no simple formula for $S$ is available if the operators in $\mathscr{A}$ do not commute.

Note that in this special case the kernel $K(t, s)=\mathbf{1}_{\{s<t\}} A(0) e^{-\lambda(t-s)} S(t, s)$ satisfies the Calderón-Zygmund estimates of [45]. Indeed, note that $\frac{\partial K}{\partial t}=-\mathbf{1}_{\{s<t\}}(\lambda+$ $A(t)) A(0) e^{-\lambda(t-s)} S(t, s)$ and $\frac{\partial K}{\partial s}=\mathbf{1}_{\{s<t\}}(\lambda+A(s)) A(0) e^{-\lambda(t-s)} S(t, s)$. Now since for all $r \in \mathbb{R}$ and $B \in \mathscr{A},\|A(r) x\| \leq N C\left(\|x\|_{X_{0}}+\|B x\|_{X_{0}}\right)$, we find that for all $r, \tau \in \mathbb{R}$ and $s<t$ letting $\sigma=(t+s) / 2$,

$$
\begin{aligned}
\|A(r) A(\tau) S(t, s)\| & =\|A(r) S(t, \sigma)\|\|A(\tau) S(\sigma, s)\| \\
& \leq N^{2} C^{2}\left(1+\left\|A_{\sigma t} S(t, \sigma)\right\|\right)\left(1+\left\|A_{s \sigma} S(\sigma, s)\right\|\right) \\
& \leq C^{\prime}\left(1+(t-s)^{-1}\right)^{2} \leq \frac{3}{2} C^{\prime}\left(1+(t-s)^{-2}\right) .
\end{aligned}
$$

Therefore, the extrapolation results from the unweighted case to the weighted case of Remark 3.8 does hold in this situation. 
Proposition 4.5 Let $S$ be an evolution family for A. Fix $x \in X_{0}$ and $f \in L^{1}\left(a, b ; X_{0}\right)$. If Eq. 4.2 has a strong solution $u \in L^{1}\left(a, b ; X_{1}\right) \cap W^{1,1}\left(a, b ; X_{0}\right) \cap C\left([a, b] ; X_{0}\right)$, then it satisfies

$$
u(t)=S(t, s) u(s)+\int_{s}^{t} S(t, r) f(r) d r, a<s \leq t<b,
$$

where we allow $s=a$ and $t=b$ whenever these are finite numbers. In particular, strong solutions are unique if $a>-\infty$. In the case $a=-\infty$ this remains true if $\lim _{s \rightarrow-\infty}\|S(t, s)\|=0$.

A partial converse is used and proved in Theorem 4.9.

Proof Fix $a<s<t<b$. By approximation one easily checks that for $u \in$ $W^{1,1}\left(s, b ; X_{0}\right) \cap L^{1}\left(s, b ; X_{1}\right), r \mapsto S(t, r) u(r)$ is in $W^{1,1}\left(s, b ; X_{0}\right)$ and

$$
\frac{d}{d r}[S(t, r) u(r)]=-S(t, r) A(r) u(r)+S(t, r) u^{\prime}(r), \quad r \in(s, T) .
$$

Applying Eq. 4.4 to the strong solution $u$ of Eq. 4.2, yields $\frac{d}{d r}[S(t, r) u(r)]=S(t, r) f(r)$. Integrating this identity over $(s, t)$, we find Eq. 4.3.

If $a>-\infty$, then we may take $s=a$ in the above proof and hence we can replace $u(s)=u(a)$ by the initial value $x$. If $a=\infty$, the additional assumption on $S$ allows us to let $s \rightarrow-\infty$ to obtain

$$
u(t)=\int_{-\infty}^{t} S(t, r) f(r) d r, \quad t<b .
$$

Corollary 4.6 If $S_{1}$ and $S_{2}$ are both evolution families for $A$, then $S_{1}=S_{2}$.

\subsection{Assumptions on $A$}

The following condition can be interpreted as an abstract ellipticity condition.

(E) Assume that $X_{0}$ has finite cotype and assume that there exists $A_{0} \in \mathscr{L}\left(X_{1}, X_{0}\right)$ which has a bounded $H^{\infty}$-calculus of angle $\sigma<\pi / 2$ and there exists a strongly continuous evolution system $(T(t, s))_{s \leq t}$ for $\left(A(t)-A_{0}\right)_{t \in \mathbb{R}}$ such that $e^{-r A_{0}}$ commutes with $T(t, s)$ for every $t \geq s$ and $r \in \mathbb{R}_{+}$and assume there exists an $\omega \in \mathbb{R}$ such that

$$
\|T(t, s)\|_{\mathscr{L}\left(X_{0}\right)} \leq M e^{\omega(t-s)}, \quad s \leq t .
$$

Set $T(t, s)=0$ for $t<s$. The following $\mathcal{R}$-boundedness condition will be used.

(Rbdd) Assume that the family $\mathcal{I}:=\left\{I_{\omega, k T}: k \in \mathcal{K}\right\} \subseteq \mathscr{L}\left(L^{p}\left(\mathbb{R}, v, X_{0}\right)\right)$ is $\mathcal{R}$ bounded, where for $k \in \mathscr{K}$ and $f \in L^{p}\left(\mathbb{R}, v ; X_{0}\right)$,

$$
I_{\omega, k T} f(t):=\int_{\mathbb{R}} k(t-s) e^{-\omega|t-s|} T(t, s) f(s) d s .
$$

\section{Remark 4.7}

1. By (A) and (E) there is a constant $C$ such that

$$
\begin{aligned}
C^{-1}\left(\|A(t) x\|_{X_{0}}+\|x\|_{X_{0}}\right) & \leq\left\|A_{0} x\right\|_{X_{0}}+\|x\|_{X_{0}} \\
& \leq C\left(\|A(t) x\|_{X_{0}}+\|x\|_{X_{0}}\right), \quad t \in \mathbb{R}
\end{aligned}
$$

and both norms are equivalent to $\|x\|_{X_{1}}$. 
2. For $m$ even, if the $A(t)$ are $m$-th order elliptic operators with $x$-independent coefficients one typically takes $A_{0}=\delta(-\Delta)^{m}$ with $\delta>0$ small enough.

3. For $p, q \in(1, \infty), v \in A_{p}$ and $X=L^{q}$, the $\mathcal{R}$-boundedness assumption follows from the weighted boundedness of $T(t, s)$ for all $w \in A_{q}$ (see Theorem 2.6).

4. Although we allow $p=1$ and $v=1$ in the above assumptions, checking the assumption (Rbdd) seems more difficult in this limiting case.

Lemma 4.8 Under the assumptions $(A)$ and $(E)$ the evolution family $S$ for A uniquely exists and satisfies

$$
\begin{aligned}
S(t, s) & =e^{-(t-s) A_{0}} T(t, s) \\
& =T(t, s) e^{-(t-s) A_{0}}=e^{-\frac{1}{2}(t-s) A_{0}} T(t, s) e^{-\frac{1}{2}(t-s) A_{0}}, \quad s \leq t,
\end{aligned}
$$

and there is a constant $C$ such that for all $s \leq t,\|S(t, s)\|_{\mathscr{L}\left(X_{0}\right)} \leq C e^{\omega(t-s)}$. Moreover, there is a constant $C$ such that,

$$
\|S(t, s)\|_{\mathscr{L}\left(X_{1}\right)} \leq C\|S(t, s)\|_{\mathscr{L}\left(X_{0}\right)}, \quad s \leq t .
$$

Proof The second identity follows from (E). To prove the first identity, we check that $S(t, s)$ given by Eq. 4.6 is an evolution family for $A$. By Corollary 4.6 this would complete the proof. It is simple to check properties (i) and (ii) of Definition 4.2 and it remains to check (iii) and (iv). Let $x \in X_{1}$. By the product rule for weak derivatives and (E) we find

$$
\begin{aligned}
\frac{d}{d t} S(t, s) x & =-A_{0} e^{-(t-s) A_{0}} T(t, s) x-\left(A(t)-A_{0}\right) T(t, s) e^{-(t-s) A_{0}} x \\
& =-A_{0} S(t, s) x-\left(A(t)-A_{0}\right) S(t, s) x=-A(t) S(t, s) x .
\end{aligned}
$$

Similarly, one checks that $\frac{d}{d s} S(t, s) x=S(t, s) A(s) x$. The fact that $S(t, s)$ satisfies the same exponential estimate as $T(t, s)$ follows from the estimate (2.7) applied to $A_{0}$.

By assumptions, for every $x \in X_{1}, e^{-r A_{0}} S(t, s) x=S(t, s) e^{-r A_{0}} x$. Thus, by differentiation we find $-A_{0} S(t, s) x=-S(t, s) A_{0} x$ and therefore

$$
\begin{aligned}
\|S(t, s) x\|_{X_{1}} & \leq C\left(\left\|A_{0} S(t, s) x\right\|_{X_{0}}+\|S(t, s) x\|_{X_{0}}\right) \\
& \leq C\left(\left\|S(t, s) A_{0} x\right\|_{X_{0}}+\|S(t, s) x\|_{X_{0}}\right) \\
& \leq C\|S(t, s)\| \mathscr{L}\left(X_{0}\right)\left(\left\|A_{0} x\right\|_{X_{0}}+\|x\|_{X_{0}}\right) \leq C^{\prime}\|S(t, s)\| \mathscr{L}\left(X_{0}\right)\|x\|_{X_{1}} .
\end{aligned}
$$

\subsection{Main Result on Maximal $L^{p}$-Regularity}

Next we will present our main abstract result on the regularity of the strong solution to the problem

$$
u^{\prime}(t)+(A(t)+\lambda) u(t)=f(t), \quad t \in \mathbb{R}
$$

Theorem 4.9 Assume (A), (E), and (Rbdd). For any $\lambda>\omega$ and for every $f \in L^{p}\left(\mathbb{R}, v ; X_{0}\right)$ there exists a unique strong solution $u \in W^{1, p}\left(\mathbb{R}, v ; X_{0}\right) \cap L^{p}\left(\mathbb{R}, v ; X_{1}\right)$ of Eq. 4.7. Moreover, there is a constant $C$ independent of $f$ and $\lambda$ such that

$$
\begin{aligned}
(\lambda-\omega)\|u\|_{L^{p}\left(\mathbb{R}, v, X_{0}\right)}+\left\|A_{0} u\right\|_{L^{p}\left(\mathbb{R}, v ; X_{0}\right)} & \leq C\|f\|_{L^{p}\left(\mathbb{R}, v ; X_{0}\right)} \\
\left\|u^{\prime}\right\|_{L^{p}\left(\mathbb{R}, v ; X_{0}\right)} & \leq \frac{C(\lambda-\omega+1)}{\lambda-\omega}\|f\|_{L^{p}\left(\mathbb{R}, v ; X_{0}\right)} .
\end{aligned}
$$


Remark 4.10 Parts of the theorem can be extended to $\lambda=\omega$, but we will not consider this in detail. The constant in the estimate (4.8) for $u^{\prime}$ can be improved if one knows $\|A(t) x\|_{X_{0}} \leq$ $C\left\|A_{0} x\right\|_{X_{0}}$ or when taking $\lambda \geq \omega+1$ for instance.

Before we turn to the proof of Theorem 4.9 we introduce some shorthand notation. Let $S_{\lambda}(t, s)=e^{-\lambda(t-s)} S(t, s)$ and $T_{\lambda}(t, s)=e^{-\lambda(t-s)} T(t, s)$. Since by Lemma 4.8, $S$ is an evolution family for $A$, also $S_{\lambda}$ is the evolution family for $A(t)+\lambda$. Similarly, $T_{\lambda}(t, s)$ is an evolution family for $A(t)-A_{0}+\lambda$. By Eq. 4.3 if the support of $f \in L^{1}\left(\mathbb{R} ; X_{0}\right)$ is finite, a strong solution of Eq. 4.7 satisfies

$$
u(t)=\int_{-\infty}^{t} S_{\lambda}(t, r) f(r) d r, \quad t \in \mathbb{R} .
$$

Proof Replacing $A(t)$ and $T(t, s)$ by $A(t)+\omega$ and $e^{-(t-s) \omega} T(t, s)$ one sees that without loss of generality we may assume $\omega=0$ in (E) and (Rbdd). We first prove that $u$ given by Eq. 4.9, is a strong solution and Eq. 4.8 holds. First let $f \in L^{p}\left(\mathbb{R}, v ; X_{1}\right)$ and such that $f$ has support on the finite interval $[a, b]$. Later on we use a density argument for general $f \in L^{p}\left(\mathbb{R}, v ; X_{0}\right)$. Let $u$ be defined as in Eq. 4.9. Note that $u=0$ on $(-\infty, a]$.

Step 1: By Lemma 4.8 the function $u$ defined by Eq. 4.9 satisfies

$$
\begin{aligned}
\|u(t)\|_{X_{1}} & \leq \int_{-\infty}^{t}\left\|S_{\lambda}(t, s)\right\|_{\mathscr{L}\left(X_{1}\right)}\|f(s)\|_{X_{1}} d s \\
& \leq C^{\prime}\|f\|_{L^{1}\left(a, b ; X_{1}\right)} \leq C\left([v]_{A_{p}}\right)\|f\|_{L^{p}\left(\mathbb{R}, v ; X_{1}\right)} .
\end{aligned}
$$

We show that $u$ is a strong solution of Eq. 4.2. Observe that from Fubini's Theorem and $\frac{d}{d s} S_{\lambda}(s, r) x=-(\lambda+A(s)) S_{\lambda}(s, r) x$ for $x \in X_{1}$, we deduce

$$
\begin{aligned}
\int_{-\infty}^{t}(\lambda+A(s)) u(s) d s & =\int_{-\infty}^{t} \int_{-\infty}^{s}(\lambda+A(s)) S_{\lambda}(s, r) f(r) d r d s \\
& =\int_{-\infty}^{t} \int_{r}^{t}(\lambda+A(s)) S_{\lambda}(s, r) f(r) d s d r \\
& =\int_{-\infty}^{t}\left(-S_{\lambda}(t, r) f(r)+f(r)\right) d r=-u(t)+\int_{-\infty}^{t} f(r) d r .
\end{aligned}
$$

Therefore, $u$ is a strong solution of Eq. 4.7.

Step 2: In this step we show there exists a $C \geq 0$ independent of $\lambda$ and $f$ such that

$$
\left\|A_{0} u\right\|_{L^{p}\left(\mathbb{R}, v ; X_{0}\right)} \leq C\|f\|_{L^{p}\left(\mathbb{R}, v ; X_{0}\right)} .
$$

By Eqs. 4.6 and 4.9 we can write $A_{0} u=I_{K} f$, where

$$
K(t, s)=\frac{\phi\left((t-s) A_{0}\right) T_{\lambda}(t, s) \phi\left((t-s) A_{0}\right)}{t-s} .
$$

Here $\phi \in H_{0}^{\infty}\left(\Sigma_{\sigma^{\prime}}\right)$ for $\sigma^{\prime}<\pi / 2$ is given by $\phi(z)=z^{1 / 2} e^{-z / 2}$. In order to apply Theorem 3.4, we note that all assumptions (H1)-(H4) are satisfied. Only the $\mathcal{R}$-boundedness condition (H4) requires some comment. Note that $k \in \mathcal{K}$ implies that for all $\lambda \geq 0, k_{\lambda} \in \mathcal{K}$ where $k_{\lambda}(t)=e^{-\lambda t} \mathbf{1}_{\{t>0\}} k(t)$. Therefore, it follows from (Rbdd) that for all $\lambda \geq 0$,

$$
\mathcal{R}\left(I_{k T_{\lambda}}: k \in \mathcal{K}\right)=\mathcal{R}\left(I_{k_{\lambda} T}: k \in \mathcal{K}\right) \leq \mathcal{R}\left(I_{k T}: k \in \mathcal{K}\right)<\infty
$$

which gives (H4) with a uniform estimate in $\lambda$. Now Eq. 4.10 follows from Theorem 3.4. 
Step 3: In this step we show there exists a $C \geq 0$ independent of $\lambda$ and $f$ such that

$$
\lambda\|u\|_{L^{p}\left(\mathbb{R}, v ; X_{0}\right)} \leq C\|f\|_{L^{p}\left(\mathbb{R}, v ; X_{0}\right)} .
$$

Using Eq. 4.9 and $\|S(t, s)\| \leq C$ we find

$$
\lambda\|u\|_{X_{0}} \leq \int_{-\infty}^{t} \lambda\left\|S_{\lambda}(t, s) f(s)\right\|_{X_{0}} d s \leq C \int_{-\infty}^{t} \lambda e^{-\lambda(t-s)}\|f(s)\|_{X_{0}} d s \leq C r_{\lambda} * g(t),
$$

where $r_{\lambda}(t)=\lambda e^{-\lambda|t|}$ and $g(s)=\|f(s)\|_{X_{0}}$ As $r_{1} \in L^{1}(\mathbb{R})$ is radially decreasing by [37, Theorem 2.1.10] and [38, Theorem 9.1.9],

$$
\begin{aligned}
\lambda\|u\|_{L^{p}\left(\mathbb{R}_{+}, v ; X_{0}\right)} & \leq C\left\|r_{\lambda} * g\right\|_{L^{p}(\mathbb{R}, v)} \\
& \leq C\|M g\|_{L^{p}(\mathbb{R}, v)} \leq C^{\prime}\|g\|_{L^{p}(\mathbb{R}, v)}=C^{\prime}\|f\|_{L^{p}\left(\mathbb{R}, v ; X_{0}\right)}
\end{aligned}
$$

in the case $p>1$. The case $p=1$ follows from Fubini's theorem and the convention $v \equiv 1$. This estimate yields (4.11).

Step 4: To prove the estimate for $u^{\prime}$ note that $u^{\prime}=-\lambda u-A u+f$, and hence writing $Z=L^{p}\left(\mathbb{R}, v ; X_{0}\right)$, by Eqs. 4.5 and 4.8 , we obtain

$$
\begin{aligned}
\left\|u^{\prime}\right\|_{Z} & \leq \lambda\|u\|_{Z}+\|A u\|_{Z}+\|f\|_{Z} \\
& \leq(\lambda+C)\|u\|_{Z}+C\left\|A_{0} u\right\|_{Z}+\|f\|_{Z} \leq K\left(\frac{\lambda+C}{\lambda-\omega}+1\right)\|f\|_{Z} .
\end{aligned}
$$

This finishes the proof of Eq. 4.8 for $f \in L^{p}\left(\mathbb{R} ; X_{1}\right)$ with support in $[a, b]$

Step 5: Now let $f \in L^{p}\left(\mathbb{R}, v ; X_{0}\right)$. Choose for $n \geq 1, f_{n} \in L^{p}\left(\mathbb{R}, v ; X_{1}\right)$ with compact support and such that $f_{n} \rightarrow f$ in $L^{p}\left(\mathbb{R}, v ; X_{0}\right)$. For each $n \geq 1$ let $u_{n}$ be the corresponding strong solution of Eq. 4.7 with $f$ replaced by $f_{n}$. From Eq. 4.8 applied to $u_{n}-u_{m}$ we can deduce that $\left(u_{n}\right)_{n \geq 1}$ is a Cauchy sequence and hence convergent to some $\bar{u}$ in $L^{p}\left(\mathbb{R}, v ; X_{1}\right) \cap W^{1, p}\left(\mathbb{R}, v ; X_{0}\right)$. On the other hand, for $u$ defined as in Eq. 4.9 one can show in the same way as in Step 3 that for almost all $t \in \mathbb{R}$,

$$
\begin{aligned}
\left\|u(t)-u_{n}(t)\right\| & \leq \int_{-\infty}^{t}\left\|S_{\lambda}(t, s)\right\|\left\|f(s)-f_{n}(s)\right\| d s \\
& \leq C \int_{-\infty}^{t} e^{-\lambda(t-s)}\left\|f(s)-f_{n}(s)\right\| d s \leq C M\left(\left\|f-f_{n}\right\|\right)(t),
\end{aligned}
$$

where $M$ is the Hardy-Littlewood maximal operator. Taking $L^{p}(v)$-norms and using the boundedness of the maximal operator we find $u_{n} \rightarrow u$ in $L^{p}\left(\mathbb{R}, v ; X_{0}\right)$ and hence $u=\bar{u}$ if $p \in(1, \infty)$. Taking limits (along a subsequence), Eqs. 4.7 and 4.8 follow if $p \in(1, \infty)$. The case $p=1$ is proved similarly using Young's inequality.

It will be convenient to restate our results in terms of maximal $L_{v}^{p}$-regularity. For $-\infty \leq$ $a<b \leq \infty$, let

$$
\operatorname{MR}^{p}((a, b), v)=W^{1, p}\left((a, b), v ; X_{0}\right) \cap L^{p}\left((a, b), v ; X_{1}\right) .
$$

Definition 4.11 Let $-\infty \leq a<b \leq \infty$. Assume (A) holds and let $p \in[1, \infty)$ and $v \in A_{p}$ with the convention that $v \equiv 1$ if $p=1$. The operator-valued function $A$ is said to have maximal $L_{v}^{p}$-regularity on $(a, b)$ if for all $f \in L^{p}\left((a, b), v ; X_{0}\right)$, the problem

$$
\left\{\begin{array}{l}
u^{\prime}(t)+A(t) u(t)=f(t), \quad t \in(a, b) \\
u(a)=0,
\end{array}\right.
$$


has a unique strong solution $u:(a, b) \rightarrow X_{0}$ and there is a constant $C$ independent of $f$ such that

$$
\|u\|_{M R^{p}((a, b), v)} \leq C\|f\|_{L^{p}\left((a, b), v ; X_{0}\right)} .
$$

Here we omit the condition $u(a)=0$ if $a=-\infty$.

Of course, the reverse estimate of Eq. 4.13 holds trivially. Note that maximal $L_{v}^{p}$ regularity on $(a, b)$ implies maximal $L_{v}^{p}$-regularity on $(c, d) \subseteq(a, b)$. It is also easy to check that if $|b-a|<\infty$, the maximal $L_{v}^{p}$-regularity on $(a, b)$ for $A$ and $\lambda+A$ are equivalent. Indeed, the solutions of $u^{\prime}(t)+(\lambda+A(t)) u(t)=f(t)$ and $w^{\prime}(t)+A(t) w(t)=e^{\lambda t} f(t)$ are connected by the identity $u(t)=e^{-\lambda t} w(t)$.

The result of Theorem 4.9 immediately implies that

Corollary 4.12 Assume (A), (E) and (Rbdd). For any $\lambda>\omega, \lambda+A$ has maximal $L_{v}^{p}$ regularity on $\mathbb{R}$.

Actually the constant in the estimate can be taken uniformly in $\lambda$. Indeed, for fixed $\lambda_{0}>$ $\omega$ by Eq. 4.8 and Remark 4.10, there is a constant $C$ such that for all $\lambda \geq \lambda_{0}$ and for all $f \in L^{p}\left(\mathbb{R}_{+}, v ; X_{0}\right)$,

$$
\|u\|_{\mathrm{MR}^{p}(\mathbb{R}, v)} \leq C\|f\|_{L^{p}\left(\mathbb{R}, v ; X_{0}\right)} .
$$

This is a maximal regularity estimate with constant which is uniform in $\lambda$.

Remark 4.13 If $A$ is time independent and has an $H^{\infty}$-calculus of angle $<\pi / 2$, then setting $A_{0}=A$, and $T(t, s)=I$, Theorem 4.9 yields a maximal regularity result for autonomous equations. There are much more suitable ways to derive maximal $L^{p}$-regularity results in the autonomous case (see $[56,62,91,92]$ ), using less properties of the operator $A$. Indeed, only $\mathcal{R}$-sectoriality of $A$ is needed, but the Banach space $X_{0}$ is assumed to be a UMD space. We assume more on the operator but less on the space as we only require finite cotype of $X_{0}$ and the $\mathcal{R}$-boundedness of a certain integral operator. Another theory where no assumptions on the Banach space are made but even more on the operator, can be found in [52]. In the above mentioned works only maximal $L^{p}$-regularity on $\mathbb{R}_{+}$is considered, but by a standard trick due to Kato one can always reduce to this case (see for instance the proof of [27, Theorem 7.1]). For the case of time-dependent operators this is no longer true.

\subsection{Traces and Initial Values}

Recall from Lemma 4.1 that any $u \in W^{1, p}\left((a, b), v ; X_{0}\right)$ has a continuous version. We introduce certain interpolation spaces in order to give a more precise description of traces. Let $X_{v, p}$ be the space of all $x \in X_{0}$ for which there is a $u \in \operatorname{MR}^{p}\left(\mathbb{R}_{+}, v\right)$ such that $u(0)=x$. Let

$$
\|x\|_{X_{v, p}}=\inf \left\{\|u\|_{\mathrm{MR}^{p}\left(\mathbb{R}_{+}, v\right)}: u(0)=x\right\} .
$$

Spaces of this type have been studied in the literature (see $[10,12,51]$ and references therein). Obviously, one has $X_{1} \hookrightarrow X_{v, p} \hookrightarrow X_{0}$.

For $t \in \mathbb{R}$ and a weight $v$, let $v_{t}=v(\cdot-t)$. The following trace estimate on $\mathbb{R}_{+}$is a direct consequence of the definitions. A similar assertions holds for $u \in \operatorname{MR}^{p}(\mathbb{R}, v)$ for all $t \in \mathbb{R}$.

Proposition 4.14 (Trace estimate) For $u \in M R^{p}\left(\mathbb{R}_{+}, v\right)$, one has

$$
\|u(t)\|_{X_{v_{t}, p}} \leq\|u\|_{M R^{p}\left(\mathbb{R}_{+}, v\right)}, \quad t \in[0, \infty) .
$$


A simple application of maximal regularity is that one can automatically consider nonzero initial values. Note that without loss of generality we can let $a=0$.

Proposition 4.15 Assume $(A)$ and let $T \in(0, \infty]$. Assume A has maximal $L_{v}^{p}$-regularity on $(0, T)$ with constant $K_{A}$. For $x \in X_{0}$ and $f:(0, T) \rightarrow X_{0}$ strongly measurable the following are equivalent:

(1) The data satisfies $x \in X_{v, p}$ and $f \in L^{p}\left((0, T), v ; X_{0}\right)$

(2) There exists a unique strong solution $u \in M R^{p}((0, T), v)$ of

$$
\left\{\begin{array}{l}
u^{\prime}(t)+A(t) u(t)=f(t), \quad t \in(0, T) \\
u(0)=x
\end{array}\right.
$$

In this case there is a constant $c_{v, p, T}$ such that the following estimate holds:

$$
\begin{aligned}
\max \left\{c_{v, p, T}\|x\|_{X_{v, p}},\|f\|_{L^{p}\left((0, T), v ; X_{0}\right)}\right\} & \leq\|u\|_{M R^{p}((0, T), v)} \\
& \leq K_{A}\|x\|_{X_{v, p}}+K_{A}\|f\|_{L^{p}\left((0, T), v ; X_{0}\right)} .
\end{aligned}
$$

Proof $(1) \Rightarrow(2)$ : Let $w \in \operatorname{MR}^{p}\left(\mathbb{R}_{+}, v\right)$ be such that $w(0)=x$. Let $g(t)=-\left(w^{\prime}(t)+\right.$ $A(t) w(t))$. Then $g \in L^{p}\left((0, T), v ; X_{0}\right)$. Let $\tilde{u}$ be the solution to Eq. 4.16 with zero initial value and with $f$ replaced by $f+g$. Now $u(t)=\tilde{u}(t)+w(t)$ is the required strong solution of Eq. 4.16. Indeed, clearly $u(0)=x$ and

$$
\begin{aligned}
u^{\prime}(t)+A(t) u(t) & =\tilde{u}^{\prime}(t)+A(t) \tilde{u}(t)+w^{\prime}(t)+A(t) w(t) \\
& =f+g-g=f .
\end{aligned}
$$

Moreover,

$$
\begin{aligned}
& \|u\|_{\mathrm{MR}^{p}((0, T), v)} \leq\|\tilde{u}\|_{\mathrm{MR}^{p}((0, T), v)}+\|w\|_{\mathrm{MR}^{p}((0, T), v)} \\
& \leq K_{A}\|f\|_{L^{p}\left((0, T), v ; X_{0}\right)}+K_{A}\|w\|_{\mathrm{MR}^{p}\left(\mathbb{R}_{+}, v\right)} .
\end{aligned}
$$

Taking the infimum over all $w \in \mathrm{MR}^{p}\left(\mathbb{R}_{+}, v\right)$ with $w(0)=x$ also yields the second part of Eq. 4.17 .

$(2) \Rightarrow(1)$ : As $u^{\prime}$ and $A u$ are both in $L^{p}\left((0, T), v ; X_{0}\right)$, the identity in Eq. 4.16 yields that $f \in L^{p}\left((0, T), v ; X_{0}\right)$ with the estimate as stated. To obtain the required properties for $x$ note that $u \in \operatorname{MR}^{p}((0, T), v)$ can be extended to a function $u \in \mathrm{MR}^{p}\left(\mathbb{R}_{+}, v\right)$ with $c_{v, p, T}\|u\|_{\mathrm{MR}^{p}\left(\mathbb{R}_{+}, v\right)} \leq\|u\|_{\mathrm{MR}^{p}((0, T), v)}$. In the case $T=\infty$ we can take $c_{v, p, T}=1$.

It can be difficult to identify $X_{v, p}$. For power weights this is possible. Including a power weight has become an important standard technique to allow non-smooth initial data and to create compactness properties. At the same time, the regularity properties of the solution to Eq. 4.16 for $t>0$ are unchanged. For more details and applications to evolution equations we refer to $[39,59,67,72,73,83]$.

Example 4.16 Assume $v(t)=t^{\alpha}$ with $\alpha \in(-1, p-1)$. Then $v \in A_{p}$ and $X_{v, p}=$ $\left(X_{0}, X_{1}\right)_{1-\frac{1+\alpha}{p}, p}$ (see [90, Theorem 1.8.2]). Here $\left(X_{0}, X_{1}\right)_{\theta, p}$ stands for the real interpolation space between $X_{0}$ and $X_{1}$. In the limiting cases $\alpha \uparrow p-1$ and $\alpha \downarrow-1$, one sees that the endpoint $X_{1}$ and $X_{0}$ can almost be reached.

As in [83] we find that for $\alpha \in[0, p-1)$, any $u \in \mathrm{MR}^{p}\left(\mathbb{R}_{+}, v\right)$ has a continuous version with values in $\left(X_{0}, X_{1}\right)_{1-\frac{1+\alpha}{p}, p}$ and

$$
\sup _{t \in \mathbb{R}_{+}}\|u(t)\|_{\left(X_{0}, X_{1}\right)_{1-\frac{1+\alpha}{p}, p}} \leq C\|u\|_{\mathrm{MR}^{p}\left(\mathbb{R}_{+}, v\right)} .
$$


Indeed, this follows from the boundedness and strong continuity of the left-translation in $L^{p}\left(\mathbb{R}_{+}, v ;\left(X_{0}, X_{1}\right)_{1-\frac{1+\alpha}{p}, p}\right)$ and Proposition 4.14.

On the other hand, for every $-1<\alpha<p-1$ one has $u \in C\left((0, \infty) ;\left(X_{0}, X_{1}\right)_{1-\frac{1}{p}, p}\right)$ and for every $\varepsilon>0$,

$$
\sup _{t \in[\varepsilon, \infty)} t^{\alpha / p}\|u(t)\|_{\left(X_{0}, X_{1}\right)_{1-\frac{1}{p}, p}} \leq C\left\|t \mapsto t^{\alpha / p} u(t)\right\|_{\mathrm{MR}^{p}(\varepsilon, \infty)} \leq C_{\varepsilon}\|u\|_{\mathrm{MR}^{p}\left(\mathbb{R}_{+}, v\right)},
$$

where we used $t^{-p} \leq \max \left\{1, \varepsilon^{-p}\right\}$. If additionally $u(0)=0$, then by Hardy's inequality (see [46, p. 245-246]) we can take $\varepsilon=0$ in the last estimate.

Proof of Theorem 1.1 First of all we may use a constant extension of $A$ to an operator family on $\mathbb{R}$. Clearly, we can do this in such a way that $T(t, s)$ is uniformly bounded in $-\infty<s \leq t<\infty$ say by a constant $M$. For instance one can take $A(t)=A_{0}$ for $t \notin(0, \tau)$. Assumption (A) is clearly satisfied. Note that by the assumption and [62, Theorem 11.13], $A_{0}$ has a bounded $H^{\infty}$-calculus of angle $<\pi / 2$ and hence $(\mathrm{E})$ is satisfied.

By Proposition $2.4\left\{I_{k T}: k \in \mathcal{K}\right\}$ is uniformly bounded. For $p=2$, this implies $\mathcal{R}$ boundedness of $\left\{I_{k T}: k \in \mathcal{K}\right\} \subseteq \mathscr{L}\left(L^{2}\left(\mathbb{R}, v ; X_{0}\right)\right)$, because $L^{2}\left(\mathbb{R}, v ; X_{0}\right)$ is a Hilbert space. By Proposition 2.5 this implies that $\left\{I_{k T}: k \in \mathcal{K}\right\} \subseteq \mathscr{L}\left(L^{p}\left(\mathbb{R}, v ; X_{0}\right)\right)$ is $\mathcal{R}$-bounded as well and hence condition (Rbdd) holds. Therefore, all the conditions of Theorem 4.9 are satisfied, and we find that $A$ has maximal $L_{v}^{p}$-regularity on $\mathbb{R}$. This implies that $A$ has maximal $L_{v}^{p}$-regularity on $(0, \tau)$, and hence the required result follows from Proposition 4.15 and Example 4.16.

\subsection{Perturbation and Approximation}

In this section we will illustrate how the additional parameter $\lambda$ from Eq. 4.14 can be used to solve the perturbed problem

$$
\left\{\begin{array}{l}
u^{\prime}(t)+A(t) u(t)+B(t, u(t))=f(t), t \in(0, T) \\
u(0)=x
\end{array}\right.
$$

Here $B:[0, T] \times X_{1} \rightarrow X_{0}$ is such that there exists a constant $\varepsilon>0$ small enough and constants $C, L \geq 0$ such that for all $x, y \in X_{1}$ and $t \in(0, T)$,

$$
\begin{aligned}
\|B(t, x)-B(t, y)\|_{X_{0}} & \leq \varepsilon\|x-y\|_{X_{1}}+L_{B}\|x-y\|_{X_{0}}, \\
\|B(t, x)\|_{X_{0}} & \leq C_{B}\left(1+\|x\|_{X_{1}}\right) .
\end{aligned}
$$

Recall that $\operatorname{MR}^{p}((0, T), v)=W^{1, p}\left((0, T), v ; X_{0}\right) \cap L^{p}\left((0, T), v ; X_{1}\right)$.

Proposition 4.17 Assume $T<\infty$. Assume (A) holds and assume there is a $\lambda_{0}$ such that for all $\lambda \geq \lambda_{0}, \lambda+A$ has maximal $L_{v}^{p}$-regularity on $(0, T)$ and there is a constant $C_{A}>0$ such that for all $\lambda \geq \lambda_{0}$ and $f \in L^{p}\left((0, T), v ; X_{0}\right)$, the strong solution $u$ to Eq. 4.12 satisfies

$$
\lambda\|u\|_{L^{p}\left((0, T), v ; X_{0}\right)}+\|u\|_{M R^{p}((0, T), v)} \leq C_{A}\|f\|_{L^{p}\left((0, T), v ; X_{0}\right)} .
$$

Assume the constant from Eq. 4.20 satisfies $\varepsilon<\frac{1}{C_{A}}$. Then for every $f \in L^{p}\left((0, T), v ; X_{0}\right)$ and $x \in X_{v, p}$, there exists a unique strong solution $u \in M R^{p}((0, T), v)$ of Eq. 4.19 and

$$
\|u\|_{M R^{p}((0, T), v)} \leq C\left(1+\|x\|_{X_{v, p}}+\|f\|_{L^{p}\left((0, T), v ; X_{0}\right)}\right),
$$

where $C$ is independent of $f$ and $x$. 
The proof of this proposition is a standard application of the regularity estimate (4.21) combined with the Banach fixed point theorem. A similar result holds on infinite time intervals if one assumes $\|B(t, x)\|_{X_{0}} \leq C_{B}\|x\|_{X_{1}}$.

Proof Let $\lambda>0$ be so large that $\frac{C_{A} L_{B}}{\lambda}<C_{A} \varepsilon:=1-\theta$ and define the following equivalent norm on $\operatorname{MR}^{p}((0, T), v)$ :

$$
\|u\|_{\lambda}=\lambda\|u\|_{L^{p}\left((0, T), v ; X_{0}\right)}+\|u\|_{\mathrm{MR}^{p}((0, T), v)} .
$$

We will prove that for all $g \in L^{p}\left((0, T), v ; X_{0}\right)$ and $x \in X_{v, p}$ there exists a unique strong solution $w \in \mathrm{MR}^{p}((0, T), v)$ of

$$
w^{\prime}(t)+(A(t)+\lambda) w(t)+\tilde{B}(t, w(t))=g(t), \quad w(0)=x .
$$

and that $w$ satisfies the estimate (4.22) with $(u, f)$ replaced by $(w, g)$. Here $\tilde{B}(t, x)=$ $e^{-\lambda t} B\left(t, e^{\lambda t} x\right)$ and note that $\tilde{B}$ satisfies the same Lipschitz estimate (4.20) as $B$. To see that the required result for Eq. 4.19 follows from this, note that there is a one-to-one correspondence between both problems given by $u(t)=e^{\lambda t} w(t)$ and $f=e^{\lambda t} g$. Therefore, from now it suffices to consider Eq. 4.23.

In order to solve Eq. 4.23 we use the maximal regularity estimate Eq. 4.21 combined with Proposition 4.15 and the special choice of $\lambda$. For $\phi \in \operatorname{MR}^{p}((0, T), v)$ we write $w=L(\phi)$, where $w \in \operatorname{MR}^{p}((0, T), v)$ is the unique strong solution of

$$
w^{\prime}(t)+(A(t)+\lambda) w(t)=g(t)-\tilde{B}(t, \phi(t)), \quad w(0)=x .
$$

Then for $\phi_{1}, \phi_{2} \in \operatorname{MR}^{p}((0, T), v)$, by Eq. 4.21 one has

$$
\begin{aligned}
\left\|L\left(\phi_{1}\right)-L\left(\phi_{2}\right)\right\|_{\lambda} & \leq C_{A}\left\|\tilde{B}\left(\cdot, \phi_{1}\right)-\tilde{B}\left(\cdot, \phi_{2}\right)\right\|_{L^{p}\left((0, T), v ; X_{0}\right)} \\
& \leq C_{A} \varepsilon\left\|\phi_{1}-\phi_{2}\right\|_{L^{p}\left((0, T), v ; X_{1}\right)}+C_{A} L_{B}\left\|\phi_{1}-\phi_{2}\right\|_{L^{p}\left((0, T), v ; X_{0}\right)} \\
& \leq(1-\theta)\left\|\phi_{1}-\phi_{2}\right\|_{\lambda} .
\end{aligned}
$$

Hence $L$ is a contraction on $\operatorname{MR}^{p}((0, T), v)$ with respect to the norm $\|\cdot\|_{\lambda}$. Therefore, by the Banach fixed point theorem there is a unique $w \in \operatorname{MR}^{p}((0, T), v)$ such that $L(w)=w$. It is clear that $w$ is the required strong solution of Eq. 4.23. To prove the required estimate note that by Eq. 4.21 and Proposition 4.15 one has

$$
\begin{aligned}
\|w\|_{\lambda} & =\|L(w)\|_{\lambda} \leq\|L(w)-L(0)\|_{\lambda}+\|L(0)\|_{\lambda} \\
& \leq(1-\theta)\|w\|_{\lambda}+C_{A}\left(\|g\|_{L^{p}\left((0, T), v ; X_{0}\right)}+C_{B}\right)+C\|x\|_{X_{v, p}} .
\end{aligned}
$$

Subtracting $(1-\theta)\|w\|_{\lambda}$ on both sides, and rewriting the estimate in terms of $f$ and $u$ gives the required result.

With a similar method as in Proposition 4.17 one obtains the following perturbation result which will be used in the next Section 6 .

Proposition 4.18 Assume $T<\infty$. Assume $(A)$ holds and $A(\cdot)$ has maximal $L_{v}^{p}$-regularity on $(0, T)$ and the estimate (4.13) holds with constant $C_{A}$. Let $\varepsilon<C_{A}$. If $B:[0, T] \rightarrow$ $\mathscr{L}\left(X_{1}, X_{0}\right)$ satisfies $\|B(t) x\|_{X_{0}} \leq \varepsilon\|x\|_{X_{1}}$ for all $x \in X_{1}$ and $t \in[0, T]$, then $A+B$ has maximal $L_{v}^{p}$-regularity with constant $\frac{C_{A}}{1-C_{A} \varepsilon}$. 
Proof One can argue as in the proof of Proposition 4.17 with $\lambda=0, g=f, \tilde{B}=B$ and $1-\theta=C_{A} \varepsilon$. Moreover, if $w=L(w)$, then

$$
\begin{aligned}
\|w\|_{\mathrm{MR}^{p}((0, T), v)} & =\|L(w)-L(0)\|_{\mathrm{MR}^{p}((0, T), v)}+\|L(0)\|_{\mathrm{MR}^{p}((0, T), v)} \\
& \leq(1-\theta)\|w\|_{\mathrm{MR}^{p}((0, T), v)}+C_{A}\|f\|_{L^{p}\left((0, T), v ; X_{0}\right)},
\end{aligned}
$$

and the required estimate result follows.

Consider the sequence of problems:

$$
\left\{\begin{array}{l}
u_{n}^{\prime}(t)+A_{n}(t) u(t)=f_{n}(t), t \in(a, b) \\
u(a)=x_{n} .
\end{array}\right.
$$

Here we omit the initial condition if $a=-\infty$.

Recall that $v_{a}=v(\cdot-a)$. The following approximation result holds.

Proposition 4.19 Assume (A) holds for $A$ and $A_{n}$ for $n \geq 1$ with uniform estimates in $n$. Assume $A$ and $A_{n}$ for $n \geq 1$ have maximal $L_{v}^{p}$-regularity on $(a, b)$ with uniform estimates in $n$. Let $f_{n}, f \in L^{p}\left((a, b), v ; X_{0}\right)$ and $x_{n}, x \in X_{v_{a}, p}$ for $n \geq 1$. Then if $u$ and $u_{n}$ are the solutions to Eqs. 4.2 and 4.25 respectively, then there is a constant $C$ only dependent on the maximal $L_{v}^{p}$ regularity constants and the constants in $(A)$ such that

$$
\begin{aligned}
\left\|u_{n}-u\right\|_{M R^{p}((a, b), v) \leq C\left[\left\|x_{n}-x\right\|_{X_{v_{a}, p}}\right.} & +\left\|f_{n}-f\right\|_{L^{p}\left((a, b), v ; X_{0}\right)} \\
& \left.+\left\|\left(A_{n}-A\right) u\right\|_{L^{p}\left((a, b), v ; X_{0}\right)}\right] .
\end{aligned}
$$

In particular if $x_{n} \rightarrow x$ in $X_{v_{a}, p}$, for all $z \in X_{1}, A_{n}(t) z \rightarrow A(t) z$ in $X_{0}$ a.e. and $f_{n} \rightarrow f$ in $L^{p}\left((a, b), v ; X_{0}\right)$, then $u_{n} \rightarrow u$ in $\operatorname{MR}^{p}((a, b), v)$.

Typically, one can take $A_{n}=\varphi_{n} * A$ where $\left(\varphi_{n}\right)_{n \geq 1}$ is an approximation of the identity. If $\varphi_{n}$ are smooth functions, then $A_{n}$ will also be smooth and therefore, $A_{n}$ will generate an evolution system with many additional properties (see $[67,88]$ ).

Proof The last assertion follows from Eq. 4.26 and the dominated convergence theorem. To prove the estimate (4.26) note that $w_{n}=u_{n}-u$ satisfies the following equation

$$
w_{n}^{\prime}+A_{n} w_{n}=\left(f_{n}-f\right)+\left(A_{n}-A\right) u, \quad w_{n}(a)=x_{n}-x .
$$

Therefore, the Eq. 4.26 follows immediately from the maximal $L_{v}^{p}$-regularity estimate.

\section{An Example: $m$-th Order Elliptic Operators}

In this section let $p, q \in(1, \infty), m \in\{1,2, \ldots\}$ and consider the usual multi-index notation $D^{\alpha}=D_{1}^{\alpha_{1}} \cdot \ldots \cdot D_{d}^{\alpha_{d}}, \xi^{\alpha}=\left(\xi^{1}\right)^{\alpha_{1}} \cdot \ldots \cdot\left(\xi^{d}\right)^{\alpha_{d}}$ and $|\alpha|=\alpha_{1}+\cdots+\alpha_{d}$ for a multi-index $\alpha=\left(\alpha_{1}, \cdots, \alpha_{d}\right) \in \mathbb{N}_{0}^{d}$. Below we let $X_{0}=L^{q}\left(\mathbb{R}^{d}, w\right)$ and $X_{1}=W^{m, q}\left(\mathbb{R}^{d}, w\right)$.

Recall that $f \in W^{m, q}\left(\mathbb{R}^{d}, w\right)$ if $f \in L^{q}\left(\mathbb{R}^{d}, w\right)$ and for all $|\alpha| \leq m,\left\|D^{\alpha} f\right\|_{L^{q}\left(\mathbb{R}^{d}, w\right)}<$ $\infty$. In this case we let

$$
[f]_{W^{m, q}\left(\mathbb{R}^{d}, w\right)}=\sum_{|\alpha|=m}\left\|D^{\alpha} f\right\|_{L^{q}\left(\mathbb{R}^{d}, w\right)}, \quad\|f\|_{W^{m, q}\left(\mathbb{R}^{d}, w\right)}=\sum_{|\alpha| \leq m}\left\|D^{\alpha} f\right\|_{L^{q}\left(\mathbb{R}^{d}, w\right)} .
$$

The weights in space will be used in combination with Theorem 2.6 to obtain $\mathcal{R}$ boundedness of the integrals operators arising in (Rbdd). 
Consider an $m$-th order elliptic differential operator $A$ given by

$$
(A(t) u)(t, x):=\sum_{|\alpha| \leq m} a_{\alpha}(t, x) D^{\alpha} u(t, x), \quad t \in \mathbb{R}_{+}, x \in \mathbb{R}^{d},
$$

where $D_{j}:=-i \frac{\partial}{\partial_{j}}$ and $a_{\alpha}: \mathbb{R}_{+} \times \mathbb{R}^{d} \rightarrow \mathbb{C}$.

In this section we will give conditions under which there holds maximal $L_{v}^{p}$-regularity for $A$ or equivalently we will prove optimal $L_{v}^{p}$-regularity results for the solution to the problem

$$
\left\{\begin{array}{l}
u^{\prime}(t, x)+(\lambda+A(t)) u(t, x)=f(t, x), t \in(a, b), x \in \mathbb{R}^{d} \\
u(a, x)=u_{0}(x), \quad x \in \mathbb{R}^{d} .
\end{array}\right.
$$

A function $u$ will be called a strong $L_{v}^{p}\left(L_{w}^{q}\right)$-solution of Eq. 5.2 if $u \in \operatorname{MR}^{p}((a, b), v)$ and Eq. 5.2 holds almost everywhere.

With slight abuse of notation we write $A$ for the realization of $A$ on $X_{0}=L^{q}\left(\mathbb{R}^{d}, w\right)$ with domain $D(A)=X_{1}$. In this way (5.2) can be modeled as a problem of the form (4.16). Also, we have seen in Section 4 (and in particular Proposition 4.15) that it is more general to study maximal $L_{v}^{p}$-regularity on $\mathbb{R}$. Therefore, we will focus on this case below.

\subsection{Preliminaries on Elliptic Equations}

In this section we introduce notation and present some results for elliptic equations which will be needed below.

Let

$$
A:=\sum_{|\alpha| \leq m} a_{\alpha} D^{\alpha},
$$

with $a_{\alpha} \in \mathbb{C}$ constant. The principal symbol of $A$ is defined as

$$
A_{\sharp}(\xi):=\sum_{|\alpha|=m} a_{\alpha} \xi^{\alpha} .
$$

We say that $A$ is uniformly elliptic of angle $\theta \in(0, \pi)$ if there exists a constant $\kappa \in(0,1)$ such that

$$
A_{\sharp}(\xi) \subset \Sigma_{\theta} \text { and }\left|A_{\sharp}(\xi)\right| \geq \kappa, \quad \xi \in \mathbb{R}^{d},|\xi|=1 .
$$

If additionally there is a constant $K$ such that $\left|a_{\alpha}\right| \leq K$ for all $|\alpha| \leq m$, then we write $A \in \operatorname{Ell}(\theta, \kappa, K)$.

The following result is on the sectoriality of the operator in the $x$-independent case. The proof is an application of the Mihlin multiplier theorem.

Theorem 5.1 Let $1<q<\infty$ and $w \in A_{q}$. Assume $A \in \operatorname{Ell}\left(\theta_{0}, \kappa, K\right)$ with $\theta_{0} \in(0, \pi)$. Then for every $\theta>\theta_{0}$ there exists an $A_{q}$-consistent constant $C$ depending on the parameters $m, d, \theta_{0}-\theta, \kappa, K, q$ such that

$$
\left\|\lambda^{1-\frac{|\beta|}{m}} D^{\beta}(\lambda+A)^{-1}\right\|_{\mathscr{L}\left(L^{q}\left(\mathbb{R}^{d}, w\right)\right)} \leq C, \quad|\beta| \leq m, \lambda \in \Sigma_{\pi-\theta} .
$$

In particular, there is a constant $\tilde{C}$ depending only on $\theta$ and $C$ such that $\left\|e^{-t A}\right\| \leq \tilde{C}$.

The case of $x$-dependent coefficients can be derived by standard localization arguments, but we will not need this case below (see [43, Theorem 3.1] and [61, Section 6]). 
Proof For Eq. 5.3 we need to check that for every $\lambda \in \Sigma_{\pi-\theta}$, and $|\beta| \leq m$, the symbol $\mathcal{M}: \mathbb{R}^{d} \rightarrow \mathbb{C}$ given by

$$
\mathcal{M}(\xi)=\lambda^{1-\frac{|\beta|}{m}} \xi^{\beta}\left(\lambda+A_{\sharp}(\xi)\right)^{-1}
$$

satisfies the following: for every multiindex $\alpha \in \mathbb{N}_{0}^{d}$, there is a constant $C_{\alpha}$ which only depends on $d, \alpha, \theta-\theta_{0}, K, \kappa$ such that

$$
\left|\xi^{\alpha} D^{\alpha} \mathcal{M}(\xi)\right| \leq C_{\alpha}, \quad \xi \in \mathbb{R}^{d} .
$$

Indeed, as soon as this is checked, the result is a consequence of the weighted version of Mihlin's multiplier theorem (see [34, Theorem IV.3.9]).

In order to check the condition for $\ell \geq 0$ let $F_{\ell}$ be the span of functions of the form $\lambda^{\eta} g h^{-1}$, where $\eta \in[0,1], g: \mathbb{R}^{d} \rightarrow \mathbb{C}$ is polynomial which is homogeneous of degree $v \in \mathbb{N}_{0}$ and $h=\left(\lambda+A_{\sharp}\right)^{\mu}$ with $\mu \in \mathbb{N}$ and $\ell=m(\mu-\eta)-v$. It is clear that $\mathcal{M} \in F_{0}$. Using induction one can check that for $f \in F_{\ell}$ one has $D^{\alpha} f \in F_{\ell+|\alpha|}$.

We claim that for $f \in F_{\ell}$ the mapping $\xi \mapsto|\xi|^{\ell} f(\xi)$ is uniformly bounded. In order to prove this it suffices to consider $f=\lambda^{\eta} g h^{-1}$ with $g$ and $h$ as before, and $\ell=m(\mu-\eta)-v$. As $\xi \mapsto|\xi|^{-v} g(\xi)$ is bounded it remains to estimate

$$
\lambda^{\eta} h(\xi)^{-1}|\xi|^{\ell+v}=s^{\eta}\left(s+A_{\sharp}\left(\xi^{*}\right)\right)^{-\mu},
$$

where $\xi^{*}=\xi /|\xi|$ and $s=\lambda|\xi|^{-m}$.

Write $A_{\sharp}\left(\xi^{*}\right)=r e^{i \varphi}$ with $r=\left|A_{\sharp}\left(\xi^{*}\right)\right|$ and $|\varphi|<\theta_{0}$ and $s=\rho e^{i \psi}$ with $\rho=|s|$ and $|\psi|<\pi-\theta$. Then

$$
\left|s^{\eta}\left(s+A_{\sharp}\left(\xi^{*}\right)\right)^{-\mu}\right|=\rho^{\eta}\left|\rho e^{i \psi}+r e^{i \varphi}\right|^{-\mu}=\rho^{\eta}\left(\rho^{2}+r^{2}+2 \rho r \cos (\psi-\varphi)\right)^{-\mu / 2} .
$$

Since $\cos (\psi-\varphi) \geq \cos \left(\pi-\left(\theta-\theta_{0}\right)\right)=-\cos \left(\theta-\theta_{0}\right)=-\left(1-\varepsilon^{2}\right)$ with $\varepsilon \in(0,1)$ and $-2 \rho r \geq-\left(\rho^{2}+r^{2}\right)$ and we find

$$
\left|s^{\eta}\left(s+A_{\sharp}\left(\xi^{*}\right)\right)^{-\mu}\right| \leq \rho^{\eta}\left(\rho^{2}+r^{2}\right)^{-\mu / 2} \varepsilon^{-\mu} \leq \kappa^{\mu-\eta} \varepsilon^{-\mu},
$$

where in the last step we used $r \geq \kappa$ and $\mu \geq \eta$. This proves the claim.

In order to check (5.4) note that $\mathcal{M} \in F_{0}$ and hence by the above $D^{\alpha} \mathcal{M}(\xi) \in F_{|\alpha|}$. Therefore, the bound follows from the claim about $F_{\ell}$ and the observation that the functions $g$ arising in the linear combinations of the form $\lambda^{\eta} g h^{-1}$ satisfy $|g(\xi)| \leq C_{K, d, \alpha}$.

The assertion for $e^{-t A}$ follows from Eq. 2.7 and the estimate (5.3) with $\beta=0$.

As a consequence we obtain the following:

Corollary 5.2 Let $\lambda_{0}>0$. Under the conditions of Theorem 5.1, the operator A is closed and for every $\lambda \geq \lambda_{0}$,

$$
c\|u\|_{W^{m, q}\left(\mathbb{R}^{d}, w\right)} \leq\|(\lambda+A) u\|_{L^{q}\left(\mathbb{R}^{d}, w\right)} \leq(K+\lambda)\|u\|_{W^{m, q}\left(\mathbb{R}^{d}, w\right)},
$$

where $c^{-1}$ is $A_{q}$-consistent and only depends on $m, d, \theta_{0}-\theta, \kappa, K, q$ and $\lambda_{0}$.

Corollary 5.2 for $x$-dependent coefficients will be derived from Theorem 5.4 in Remark 5.6.

Corollary 5.3 Let $m \geq 1,1<q<\infty$ and $w \in A_{q}$. If $m \geq 2$, then there is an $A_{q}$-consistent constant $C$ depending only on $d, q$ and $m$ such that for all $|\beta| \leq m-1$

$$
\begin{aligned}
\left\|D^{\beta} f\right\|_{L^{q}\left(\mathbb{R}^{d}, w\right)} & \leq C\|f\|_{L^{q}\left(\mathbb{R}^{d}, w\right)}[f]_{W^{m, q}\left(\mathbb{R}^{d}, w\right)} \\
& \leq C^{\prime} \lambda^{\frac{\beta}{m}}\|f\|_{L^{q}\left(\mathbb{R}^{d}, w\right)}+C^{\prime} \lambda^{-\frac{m-|\beta|}{|m|}}[f]_{W^{m, q}\left(\mathbb{R}^{d}, w\right)} .
\end{aligned}
$$


Proof Note that for $|\beta|=1$,

$$
\left\|D^{\beta} f\right\|_{L^{q}\left(\mathbb{R}^{d}, w\right)} \leq C \lambda^{\frac{1}{2}}\|f\|_{L^{q}\left(\mathbb{R}^{d}, w\right)}+\lambda^{-\frac{1}{2}}[f]_{W^{2, q}\left(\mathbb{R}^{d}, w\right)}
$$

follows from Theorem 5.1 with $A=-\Delta$ and the required estimate follows by minimizing over all $\lambda>0$. The case $m>2$ can be obtained by induction (see [61, Exercise 1.5.6]). The final estimate follows from Young's inequality.

\subsection{Main Result on $\mathbb{R}^{d}$}

For $A$ of the form (5.1) and $x_{0} \in \mathbb{R}^{d}$ and $t_{0} \in \mathbb{R}$ let us introduce the notation:

$$
A\left(t_{0}, x_{0}\right):=\sum_{|\alpha| \leq m} a_{\alpha}\left(t_{0}, x_{0}\right) D^{\alpha} .
$$

for the operator with constant coefficients.

(C) Let $A$ be given by Eq. 5.1 and assume each $a_{\alpha}: \mathbb{R} \times \mathbb{R}^{d} \rightarrow \mathbb{C}$ is measurable. We assume there exist $\theta_{0} \in[0, \pi / 2), \kappa$ and $K$ such that for all $t_{0} \in \mathbb{R}$ and $x_{0} \in \mathbb{R}^{d}$, $A\left(t_{0}, x_{0}\right) \in \operatorname{Ell}\left(\theta_{0}, \kappa, K\right)$. Assume there exists an increasing function $\omega:(0, \infty) \rightarrow$ $(0, \infty)$ with the property $\omega(\varepsilon) \rightarrow 0$ as $\varepsilon \downarrow 0$ and such that

$$
\left|a_{\alpha}(t, x)-a_{\alpha}(t, y)\right| \leq \omega(|x-y|),|\alpha|=m, t \in \mathbb{R}, x, y \in \mathbb{R}^{d} .
$$

As $\theta_{0}<\pi / 2$, the above ellipticity condition implies that $m$ is even in all the results below.

The set of parameters on which all constant below will depend is given by

$$
\mathcal{P}=\left\{\kappa, K, \omega,[v]_{A_{p}},[w]_{A_{q}}, p, q, d, m, \theta_{0}\right\}
$$

Moreover, all the dependence on the weights will be in an $A_{p}$ and $A_{q}$-consistent way.

Theorem 5.4 Let $p, q \in(1, \infty)$. Let $v \in A_{p}(\mathbb{R})$ and $w \in A_{q}\left(\mathbb{R}^{d}\right)$. Assume condition $(C)$ on $A$. Then there exists a $\lambda_{0} \in \mathbb{R}$ depending on the parameters in $\mathcal{P}$ such that for all $\lambda \geq \lambda_{0}$ the operator $\lambda+A$ has maximal $L_{v}^{p}$-regularity on $\mathbb{R}$. Moreover, for every $\lambda \geq \lambda_{0}$ and for every $f \in L^{p}\left(\mathbb{R}, v ; X_{0}\right)$ there exists a unique $u \in M R^{p}(\mathbb{R}, v)$ which is a strong $L^{p}\left(L^{q}\right)$ solution of

$$
u^{\prime}(t, x)+(\lambda+A(t)) u(t, x)=f(t, x) \text {, a.e. } t \in \mathbb{R}, x \in \mathbb{R}^{d}
$$

and there is constant $C$ depending on the parameters in $\mathcal{P}$ such that

$$
\lambda\|u\|_{\left.L^{p}\left(\mathbb{R}, v ; X_{0}\right)\right)}+\|u\|_{M R^{p}(\mathbb{R}, v)} \leq C\|f\|_{L^{p}\left(\mathbb{R}, v ; X_{0}\right)} .
$$

Recall that $\operatorname{MR}^{p}(\mathbb{R}, v)=W^{1, p}\left(\mathbb{R}, v ; X_{0}\right) \cap L^{p}\left(\mathbb{R}, v ; X_{1}\right)$.

Also note that the estimate (5.6) also holds if one replaces $\mathbb{R}$ by $(-\infty, T)$ for some $T \in \mathbb{R}$. The above result also implies that $\lambda+A$ has maximal $L_{v}^{p}$-regularity on $(0, T)$ for every $T<\infty$ and every $\lambda \in \mathbb{R}$.

The proof of the above result is a based on Theorem 4.9, standard PDE techniques and extrapolation arguments. The proof of Theorem 5.4 is divided in several steps of which some are standard, but we prefer to give a complete proof for convenience of the reader. In Steps 1 and 2 we assume $a_{\alpha}=0$ for $|\alpha|<m$ and show how to include these lowers order terms later on. 
Step 1: Consider the case where the coefficients $a_{\alpha}: \mathbb{R} \rightarrow \mathbb{C}$ are $x$-independent. Choose $\delta>0$ small enough and set $A_{0}=\delta(-\Delta)^{m / 2}$. Note that by Corollary 5.2 $D\left(A_{0}\right)=X_{1}$. We write

$$
A(t)=\sum_{|\alpha|=m} a_{\alpha}(t) D^{\alpha}, \quad \tilde{A}(t)=A(t)-A_{0} .
$$

It is a simple exercise to see that there exist $\delta_{0}>0, \theta^{\prime} \in\left(\theta, \frac{\pi}{2}\right)$ and $\kappa^{\prime}>0$ depending on $\kappa$ and $\theta$ that for all $\delta \in\left(0, \delta_{0}\right], \tilde{A}(t) \in \operatorname{Ell}\left(\theta^{\prime}, \kappa^{\prime}, K\right)$. Therefore, each $\tilde{A}(t)$ satisfies the conditions of Theorem 5.1 with constants only depending on $\delta_{0}, \kappa, \theta, K$. The same holds for operators of the form $\tilde{A}_{a b}:=\frac{1}{b-a} \int_{a}^{b} \tilde{A}(t) d t$, where $0 \leq a<b<\infty$. Note that $\tilde{A}_{a b}$ and $\tilde{A}(t)$ are resolvent commuting and have domain $X_{1}$. Therefore, by Example 4.4 the evolution system for $\tilde{A}$ exists and is given by

$$
T(t, s)=\exp \left(-(t-s) \tilde{A}_{s t}\right), \quad 0 \leq s \leq t<\infty .
$$

Moreover, for all $\lambda>0$,

$$
\|T(t, s)\|_{\mathscr{L}\left(L^{q}\left(\mathbb{R}^{d}, w\right)\right)} \leq C, \quad 0 \leq s \leq t,
$$

where $C$ only depends on $\delta_{0}, \kappa, \theta, \theta_{0}, K, q,[w]_{A_{q}}$. Since $A_{0}$ is also resolvent commuting with $\tilde{A}_{a b}$ and $\tilde{A}(t)$, it follows from Lemma 4.8 that the evolution system generated by $A$ factorizes as

$$
S(t, s)=e^{-\frac{1}{2}(t-s) A_{0}} T(t, s) e^{-\frac{1}{2}(t-s) A_{0}}, \quad 0 \leq s \leq t<\infty .
$$

We check the hypothesis (A), (E) and (Rbdd) of Theorem 4.9. Condition (A) follows Corollary 5.2 with $\lambda=1$. For condition (E), recall from Section 2.3 that $A_{0}$ has a bounded $H^{\infty}$-calculus of angle $<\pi / 2$. Moreover, $X_{0}=L^{q}\left(\mathbb{R}^{d}\right)$ has finite cotype (see [25, Chapter 11]). Finally, (Rbdd) follows from Theorem 2.6 and Eq. 5.7. Therefore, by Theorem 4.9 we find there is a constant $C$ such that Eq. 5.6 holds for all $\lambda \geq 1$.

Step 2: Next we consider the case where the coefficients of $A$ are also $x$-dependent, but still with $a_{\alpha}=0$ for $\alpha<m$. We start with a standard freezing lemma.

Lemma 5.5 (Freezing lemma) Let $\varepsilon>0$ be such that $\omega(\varepsilon) \leq \frac{1}{2 C}$, where $C$ is the constant for Eq. 5.6 obtained in Step 1. If $u \in M R^{p}(\mathbb{R}, v)$ and for some $x_{0} \in \mathbb{R}^{d}$ for each $t \in \mathbb{R}$, $u(t, \cdot)$ has support in a ball $B\left(x_{0}, \varepsilon\right)=\left\{x:\left|x-x_{0}\right|<\varepsilon\right\}$, then for all $\lambda \geq 1$, the following estimate holds:

$$
\lambda\|u\|_{L^{p}\left(\mathbb{R}, v ; X_{0}\right)}+\|u\|_{M R^{p}(\mathbb{R}, v)} \leq 2 C\left\|(\lambda+A) u+u^{\prime}\right\|_{L^{p}\left(\mathbb{R}, v ; X_{0}\right)} .
$$

Proof Let $f:=(\lambda+A) u+u^{\prime}$ and observe that $u^{\prime}+\left(A\left(\cdot, x_{0}\right)+\lambda\right) u=f+\left(A\left(\cdot, x_{0}\right)-A\right) u$. By Eq. 5.6, we find

$$
\lambda\|u\|_{L^{p}\left(\mathbb{R}, v ; X_{0}\right)}+\|u\|_{\mathrm{MR}^{p}(\mathbb{R}, v)} \leq C\|f\|_{L^{p}\left(\mathbb{R}, v ; X_{0}\right)}+C\left\|\left(A\left(\cdot, x_{0}\right)-A\right) u\right\|_{L^{p}\left(\mathbb{R}, v ; X_{0}\right)} .
$$

Note that by the support condition on $u$ and the continuity of $x \mapsto a_{\alpha}(\cdot, x)$,

$$
\left\|\left(A\left(t, x_{0}\right)-A(t)\right) u(t)\right\|_{X_{0}} \leq \omega(\varepsilon)\|u(t)\|_{X_{1}} .
$$

Therefore, $C\left\|\left(A\left(\cdot, x_{0}\right)-A\right) u\right\|_{L^{p}\left(\mathbb{R}, v ; X_{0}\right)} \leq \frac{1}{2}\|u\|_{\mathrm{MR}^{p}(\mathbb{R}, v)}$ and hence

$$
\lambda\|u\|_{L^{p}\left(\mathbb{R}, v ; X_{0}\right)}+\|u\|_{\mathrm{MR}^{p}(\mathbb{R}, v)} \leq C\|f\|_{L^{p}\left(\mathbb{R}, v ; X_{0}\right)}+\frac{1}{2}\|u\|_{\mathrm{MR}^{p}(\mathbb{R}, v)} .
$$

and the result follows from this. 
Step 3: In this step we use a localization argument in the case $p=q$ to show that there is a constant $C$ such that for all $u \in \operatorname{MR}^{p}(\mathbb{R}, v)$,

$$
\lambda\|u\|_{L^{q}\left(\mathbb{R}, v ; X_{0}\right)}+\|u\|_{L^{q}\left(\mathbb{R}, v ; X_{1}\right)} \leq C\left\|(\lambda+A) u+u^{\prime}\right\|_{L^{q}\left(\mathbb{R}, v ; X_{0}\right)} .
$$

(a) Take a $\phi \in C^{\infty}\left(\mathbb{R}^{d}\right)$ with $\phi \geq 0,\|\phi\|_{L^{q}\left(\mathbb{R}^{d}\right)}=1$ and support in the ball $B_{\varepsilon}=\{x$ : $|x|<\varepsilon\}$ where $\varepsilon>0$ is as in Lemma 5.5. Note that

$$
\left|\nabla^{m} u(t, x)\right|^{q}=\int_{\mathbb{R}^{d}}\left|\nabla^{m} u(t, x) \phi(x-\xi)\right|^{q} d \xi .
$$

By the product rule, we can write

$$
\nabla^{m}[u(t, x) \phi(x-\xi)]=\nabla^{m} u(t, x) \cdot \phi(x-\xi)+\sum_{|\alpha| \leq m-1} c_{\alpha} D^{\alpha} u(t, x) D^{g(\alpha)} \phi(x-\xi),
$$

with $|g(\alpha)| \leq m$ and $c_{\alpha} \geq 0$. Therefore,

$$
\left|\nabla^{m} u(t, x) \cdot \phi(x-\xi)\right| \leq\left|\nabla^{m}[u(t, x) \phi(x-\xi)]+\tilde{C} \sum_{|\alpha| \leq m-1}\right| D^{\alpha} u(t, x) \mid,
$$

where used $\sum_{|\alpha| \leq m-1} c_{\alpha}\left|D^{g(\alpha)} \phi(x)\right| \leq \tilde{C}$. Taking $L^{q}(\mathbb{R}, v)$-norms on both sides gives

$$
\begin{aligned}
\left\|\nabla^{m} u\right\|_{L^{q}\left(\mathbb{R}, v ; X_{0}\right)} & =\left(\int_{\mathbb{R}^{d}}\left\|\nabla^{m} u \phi(\cdot-\xi)\right\|_{L^{q}\left(\mathbb{R} ; X_{0}\right)}^{q} d \xi\right)^{1 / q} \\
& \leq\left(\int_{\mathbb{R}^{d}}\left\|\nabla^{m}(u \phi(\cdot-\xi))\right\|_{L^{q}\left(\mathbb{R}, v ; X_{0}\right)}^{q} d \xi\right)^{1 / q}+L,
\end{aligned}
$$

where $L=\tilde{C} \sum_{|\alpha| \leq m-1}\left\|D^{\alpha} u\right\|_{L^{q}\left(\mathbb{R}, v ; X_{0}\right)}$. For each fixed $\xi$ in the case $p=q$, Lemma 5.5 applied to $x \mapsto u(t, x) \phi(x-\xi)$ yields

$$
\begin{aligned}
& \left\|\nabla^{m}(u(t) \phi)\right\|_{L^{q}\left(\mathbb{R}, v ; X_{0}\right)} \leq\left(\int_{\mathbb{R}^{d}}\left\|\nabla^{m}(u \phi(\cdot-\xi))\right\|_{L^{q}\left(\mathbb{R}, v ; X_{0}\right)}^{q} d \xi\right)^{1 / q}+L \\
& \leq C\left(\int_{\mathbb{R}^{d}}\left\|(\lambda+A)(u \phi(\cdot-\xi))+u^{\prime} \phi(\cdot-\xi)\right\|_{L^{q}\left(\mathbb{R}, v ; X_{0}\right)}^{q} d \xi\right)^{\frac{1}{q}}+L,
\end{aligned}
$$

Note that for each $\xi \in \mathbb{R}^{d}$,

$$
\begin{aligned}
(\lambda+A)(u \phi(\cdot-\xi)) & =\sum_{|\alpha|=m} a_{\alpha} D^{\alpha}[u \phi(\cdot-\xi)]+\lambda u \phi(\cdot-\xi) \\
& =(\lambda+A) u \cdot \phi(\cdot-\xi)+\sum_{|\alpha| \leq m-1} c_{\alpha} a_{\alpha} D^{\alpha} u D^{g(\alpha)} \phi(\cdot-\xi)
\end{aligned}
$$

Thus we also have

$$
\begin{aligned}
\left(\int_{\mathbb{R}^{d}}\right. & \left.\left\|(\lambda+A)(u \phi(\cdot-\xi))+u^{\prime} \phi(\cdot-\xi)\right\|_{L^{q}\left(\mathbb{R}, v ; X_{0}\right)}^{q} d \xi\right)^{\frac{1}{q}} \\
& \leq\left(\int_{\mathbb{R}^{d}}\left\|\left[(\lambda+A) u+u^{\prime}\right] \phi(\cdot-\xi)\right\|_{L^{q}\left(\mathbb{R}, v ; X_{0}\right)}^{q} d \xi\right)^{\frac{1}{q}}+K L \\
& =\left\|(\lambda+A) u+u^{\prime}\right\|_{L^{q}\left(\mathbb{R}, v ; X_{0}\right)}+K L .
\end{aligned}
$$

Combining the latter with Eqs. 5.11 and 5.12 gives

$$
\left\|\nabla^{m} u\right\|_{L^{q}\left(\mathbb{R}, v ; X_{0}\right)} \leq C\left\|(\lambda+A) u+u^{\prime}\right\|_{L^{q}\left(\mathbb{R}, v ; X_{0}\right)}+(K+1) L,
$$


where $K$ is as in condition (C). We may conclude that

$$
\|u\|_{L^{q}\left(\mathbb{R}, v ; X_{1}\right)} \leq C\left\|(\lambda+A) u+u^{\prime}\right\|_{L^{q}\left(\mathbb{R}, v ; X_{0}\right)}+C\|u\|_{L^{q}\left(\mathbb{R}, v ; W^{m-1, q}\left(\mathbb{R}^{d}, w\right)\right)} .
$$

To include the lower order terms, let

$$
B(t) u(t, x)=\sum_{|\alpha| \leq m-1} a_{\alpha}(t, x) D^{\alpha} u .
$$

By Eq. 5.13 with $f=(A+B+\lambda) u+u^{\prime}$ and the triangle inequality, we find

$$
\|u\|_{L^{q}\left(\mathbb{R}, v ; X_{1}\right)} \leq C\|f\|_{L^{q}\left(\mathbb{R}, v ; X_{0}\right)}+C(K+1)\|u\|_{L^{q}\left(\mathbb{R}, v ; W^{m-1, q}\left(\mathbb{R}^{d}, w\right)\right)} .
$$

In a similar way, one sees that for all $\lambda \geq 1$

$$
\lambda\|u\|_{L^{q}\left(\mathbb{R}, v ; X_{0}\right)} \leq C\|f\|_{L^{q}\left(\mathbb{R}, v ; X_{0}\right)}+C(K+1)\|u\|_{L^{q}\left(\mathbb{R}, v ; W^{m-1, q}\left(\mathbb{R}^{d}, w\right)\right)} .
$$

In order to obtain Eq. 5.9 from Eqs. 5.14 and 5.15 note that it follows from the interpolation inequality from Corollary 5.3 that for all $v>0$

$$
\|u\|_{W^{m-1, q}\left(\mathbb{R}^{d}, w\right)} \leq C v^{m-1}\|u\|_{L^{q}\left(\mathbb{R}^{d}, w\right)}+C v^{-1}\|u\|_{W^{m, q}\left(\mathbb{R}^{d}, w\right)} .
$$

Therefore, choosing $v$ small enough we can combine the latter with Eq. 5.15 to obtain

$$
\begin{aligned}
\lambda\|u\|_{L^{q}\left(\mathbb{R}, v ; X_{0}\right)}+\|u\|_{L^{q}\left(\mathbb{R}, v ; X_{1}\right)} \leq & C\|f\|_{L^{q}\left(\mathbb{R}, v ; X_{0}\right)} \\
& +\frac{1}{2}\|u\|_{L^{q}\left(\mathbb{R}, v ; X_{1}\right)}+C_{\nu}\|u\|_{L^{q}\left(\mathbb{R}, v ; X_{0}\right)} .
\end{aligned}
$$

Setting $\lambda_{0}=\max \left\{2 C_{\nu}, 1\right\}$, it follows that for all $\lambda \geq \lambda_{0}$,

$$
\frac{1}{2} \lambda\|u\|_{L^{q}\left(\mathbb{R}, v ; X_{0}\right)}+\frac{1}{2}\|u\|_{L^{q}\left(\mathbb{R}, v ; X_{1}\right)} \leq C\|f\|_{L^{q}\left(\mathbb{R}, v ; X_{0}\right)} .
$$

This clearly implies Eq. 5.9.

Step 4: To extrapolate the estimate from the previous step to $p \neq q$, let $u: \mathbb{R} \rightarrow X_{1}$ be a Schwartz function. Then by Eq. 5.9 we have for all $v \in A_{q}$ there exists $A_{q}$-consistent constants $\lambda_{0}, C>0$ such that for all $\lambda \geq \lambda_{0}$

$$
\left\|F_{\lambda}\right\|_{L^{q}(\mathbb{R}, v)} \leq C\left\|G_{\lambda}\right\|_{L^{q}(\mathbb{R}, v)},
$$

where $F_{\lambda}=\|u\|_{X_{1}}, G_{\lambda}=\left\|(\lambda+A) u+u^{\prime}\right\|_{X_{0}}$. Therefore, by the extrapolation result Theorem 2.1 it follows that for all $v \in A_{p}$ there exist a $A_{p}$-consistent constants $\lambda_{0}^{\prime}$ and $C^{\prime}$ such that for all $\lambda \geq \lambda_{0}^{\prime}$,

$$
\left\|F_{\lambda}\right\|_{L^{p}(\mathbb{R}, v)} \leq C^{\prime}\left\|G_{\lambda}\right\|_{L^{p}(\mathbb{R}, v)},
$$

This yields

$$
\|u\|_{L^{p}\left(\mathbb{R}, v ; X_{1}\right)} \leq C^{\prime}\left\|(\lambda+A) u+u^{\prime}\right\|_{L^{p}\left(\mathbb{R}, v ; X_{0}\right)} .
$$

Similarly, one proves the estimate for $\lambda\|u\|_{L^{p}\left(\mathbb{R}, v ; X_{0}\right)}$. As $u^{\prime}=(\lambda+A) u+u^{\prime}-(\lambda+A) u$, Eq. 5.6 with righthand side $f=(\lambda+A) u+u^{\prime}$ follows.

Step 5: Let $A$ be as in the theorem. For $s \in[0,1]$ let $A_{s}=s A+(1-s)(-\Delta)^{m / 2}$, where we recall that $m$ is even. Then $A_{s}$ satisfies condition (C) with constants $\kappa$ and $K$ replaced by $\min \{\kappa, 1\}$ and $\max \{K, 1\}$, respectively. Therefore, for all $\lambda \geq \lambda_{0}$, Eq. 5.6 holds with right-hand side $f=\left(\lambda+A_{s}\right) u+u^{\prime}$ with a constant $C$ which does not dependent on $s$. For $s=0$ for all $\lambda \geq \lambda_{0}$, for every $f \in L^{p}\left(\mathbb{R}, v ; X_{0}\right)$, one has existence and uniqueness of a strong solution $u \in \mathrm{MR}^{p}(\mathbb{R}, v)$ to $u^{\prime}+\left(\lambda+A_{s}\right) u=f$ by step 1 . Therefore, the method of continuity (see [36, Theorem 5.2]) yields existence and uniqueness of a strong solution for every $s \in[0,1]$. Taking $s=1$, the required result follows and this completes the proof of Theorem 5.4. 
Remark 5.6 In the proof of Theorem 5.4 we applied Corollary 5.2 only for the case of $x$-independent coefficients. It is rather simple to derive Corollary 5.2 with $x$-dependent coefficients from Theorem 5.4 (cf. [61, Exercise 4.3.13]). Indeed, let $A$ be $t$-independent but such that $(\mathrm{C})$ holds and let $u \in W^{m, q}\left(\mathbb{R}^{d}, w\right)$. Applying Eq. 5.6 to $\tilde{u}: \mathbb{R} \rightarrow X_{1}$ given by $\tilde{u}(t)=e^{-\mu|t|} u$ with $\mu>0$ and $v=1$, and letting $\mu \downarrow 0$, we find

$$
\lambda\|u\|_{L^{q}\left(\mathbb{R}^{d}, w\right)}+\|u\|_{W^{m, q}\left(\mathbb{R}^{d}, w\right)} \leq C\|(\lambda+A) u\|_{L^{q}\left(\mathbb{R}^{d}, w\right)} .
$$

Finally we show how to derive Theorem 1.2 from Theorem 5.4.

Proof of Theorem 1.2 By Theorem 5.4 there is a $\lambda \in \mathbb{R}$ such that $\lambda+A$ has maximal $L^{p}$ regularity on $\mathbb{R}$ and hence on $(0, T)$ as well. By the observation after Definition 4.11 this implies that $A$ has maximal $L^{p}$-regularity on $(0, T)$ and hence we can find a unique solution

$$
u \in W^{1, p}\left(0, T ; L^{q}\left(\mathbb{R}^{d}\right)\right) \cap L^{p}\left(0, T ; W^{m, q}\left(\mathbb{R}^{d}\right)\right)
$$

of Eq. 1.2 with $u_{0}=0$. By Proposition 4.15 with $v \equiv 1$, we can allow nonzero initial values $u_{0} \in X_{v, p}=\left(L^{q}\left(\mathbb{R}^{d}\right), W^{m, q}\left(\mathbb{R}^{d}\right)\right)_{1-\frac{1}{p}, p}$ (see Example 4.16). By [11, Theorem 6.2.4] or [90, Remark 2.4.2.4] this real interpolation space can be identified with $B_{q, p}^{s}\left(\mathbb{R}^{d}\right)$ with $s=m(1-1 / p)$. Finally, the fact that $u \in C\left([0, T] ; B_{q, p}^{s}\left(\mathbb{R}^{d}\right)\right)$ follows from Example 4.16 as well.

In the next remark we compare Theorems 1.2 and 5.4 to part of the literature on such equations.

\section{Remark 5.7}

(1) In the case $A$ is time-independent and $v \equiv 1$, Theorem 5.4 reduces to [43, Theorem 3.1 ] in case of scalar equations.

(2) In a series of papers by Kim and Krylov several maximal $L^{p}$-regularity results for Eq. 1.2 have been derived under VMO conditions on the coefficients. In [61, Theorem 4.3.8] the case $p=q$ and $m=2$ has been considered under the same assumptions under the coefficients. Extensions to the case $1<q \leq p<\infty$ have been given in [57] and [61, Chapter 7]. Here only VMO conditions in the space variable are required. In the $x$-independence case results for $p, q \in(1, \infty)$ can be found in $[58,60]$. For further results and references in the case $p=q$ we refer to [26].

In future works we will consider other examples and applications of the above methods:

\section{Remark 5.8}

(1) One can extend Theorem 5.4 to systems of equations. This is more complicated as the evolution family is not explicitly given in this situation. This will be addressed in future works.

(2) With standard methods one can extend the result of Theorem 5.4 to half spaces and domains. This will be presented elsewhere.

(3) The same method gives new information on stochastic maximal $L^{p}$-regularity for SPDEs with coefficients which depend on time in a measurable way. The case of continuous dependence on time was considered in [75]. 


\section{Quasilinear Evolution Equations}

In this section we illustrate how the results of the paper can be used to study nonlinear PDEs. We extend the result of [14] and [81] (see [59] for the weighted setting) to the case of time-dependent operators $A$ without continuity assumptions. Our proof slightly differs from the previous ones since we can immediately deal with the nonautonomous setting. For notational simplicity we consider the unweighted setting only.

\subsection{Abstract Setting}

Let $X_{0}$ be a Banach space and $X_{1} \hookrightarrow X_{0}$ densely, $0<T \leq T_{0}<\infty, J=[0, T]$, $J_{0}=\left[0, T_{0}\right]$ and $p \in(1, \infty)$. Let $X_{p}=\left(X_{0}, X_{1}\right)_{1-\frac{1}{p}, p}$ equipped with the norm from Eq. 4.15. Consider the quasi-linear problem

$$
\left\{\begin{array}{l}
u^{\prime}(t)+A(t, u(t)) u(t)=F(t, u(t)), t \in J \\
u(0)=x
\end{array}\right.
$$

where $x \in X_{p}$ and

- $\quad A: J_{0} \times X_{p} \rightarrow \mathcal{L}\left(X_{1}, X_{0}\right)$ is such that for each $y \in X_{1}$ and $x \in X_{p}, t \rightarrow A(t, x) y$ is strongly measurable and satisfies the following continuity condition: for each $R>0$ there is a constant $C(R)>0$ such that

$$
\left\|A\left(t, x_{1}\right) y-A\left(t, x_{2}\right) y\right\|_{X_{0}} \leq C(R)\left\|x_{1}-x_{2}\right\| X_{p}\|y\|_{X_{1}},
$$

with $t \in J_{0}, x_{1}, x_{2} \in X_{p},\left\|x_{1}\right\|_{X_{p}},\left\|x_{2}\right\|_{X_{p}} \leq R, y \in X_{1}$.

- $F: J_{0} \times X_{p} \rightarrow X_{0}$ is such that $F(\cdot, x)$ is measurable for each $x \in X_{p}, F(t, \cdot)$ is continuous for a.a. $t \in J_{0}$ and $F(\cdot, 0) \in L^{p}\left(J_{0} ; X_{0}\right)$ and $F$ satisfies the following condition on Lipschitz continuity: for each $R>0$ there is a function $\phi_{R} \in L^{p}\left(J_{0}\right)$ such that

$$
\left\|F\left(t, x_{1}\right)-F\left(t, x_{2}\right)\right\|_{X_{0}} \leq \phi_{R}(t)\left\|x_{1}-x_{2}\right\|_{X_{p}},
$$

for a.a. $t \in J_{0}, x_{1}, x_{2} \in X_{p},\left\|x_{1}\right\|_{X_{p}},\left\|x_{2}\right\|_{X_{p}} \leq R$.

Theorem 6.1 Assume the above conditions on $A$ and F. Let $x_{0} \in X_{p}$ and assume that $A\left(\cdot, x_{0}\right)$ has maximal $L^{p}$-regularity. Then there is a $T \in\left(0, T_{0}\right]$ and radius $\varepsilon>0$ both depending on $x_{0}$ such that for all $x \in B_{\varepsilon}=\left\{y \in X_{p}:\left\|y-x_{0}\right\| X_{p} \leq \varepsilon\right\}$, Eq. 6.1 admits $a$ unique solution $u \in M R(J):=W^{1, p}\left(J ; X_{0}\right) \cap L^{p}\left(J ; X_{1}\right)$. Moreover, there is a constant $C$ such that for all $x, y \in B_{\varepsilon}$ the corresponding solutions $u^{x}$ and $u^{y}$ satisfy

$$
\left\|u^{x}-u^{y}\right\|_{M R(J)} \leq C\|x-y\|_{X_{p}} .
$$

The proof will be given in Section 6.3.

\subsection{Example of a Quasilinear Second Order Equation}

Let $T_{0}>0$ and $J_{0}=\left[0, T_{0}\right]$. In this section we will give conditions under which there exists a local solution of the problem:

$$
u^{\prime}(t, x)+\sum_{|\alpha|=2} a_{\alpha}(t, x, u(t, x), \nabla u(t, x)) D^{\alpha} u(t, x)=f(t, x, u(t, x), \nabla u(t, x)),
$$

with initial value $u(0, x)=u_{0}(x), t \in J_{0}, x \in \mathbb{R}^{d}$ and where $D_{j}:=-i \frac{\partial}{\partial_{j}}$. The main new feature here is that the above functions $a_{\alpha}$ are only measurable in time. Note that possible 
lower order terms $a_{\alpha}$ can be included in $f$. We will provide an $L^{p}\left(L^{q}\right)$-theory for Eq. 6.3 under the following conditions on $p$ and $q$ :

(i) Let $X_{0}=L^{q}\left(\mathbb{R}^{d}\right), X_{1}=W^{2, q}\left(\mathbb{R}^{d}\right), X_{p}=B_{q, p}^{2\left(1-\frac{1}{p}\right)}\left(\mathbb{R}^{d}\right)$ where $p, q \in(1, \infty)$ satisfy

$$
2\left(1-\frac{1}{p}\right)-\frac{d}{q}>1 \text {. }
$$

This condition is to ensure the following continuous embedding holds (see [90, Theorem 2.8.1])

$$
B_{q, p}^{2\left(1-\frac{1}{p}\right)}\left(\mathbb{R}^{d}\right) \hookrightarrow C^{1+\delta}\left(\mathbb{R}^{d}\right), \quad \text { for all } 0<\delta<2\left(1-\frac{1}{p}\right)-\frac{d}{q}-1 .
$$

Also note that $B_{q, p}^{2\left(1-\frac{1}{p}\right)}\left(\mathbb{R}^{d}\right)=\left(X_{0}, X_{1}\right)_{1-\frac{1}{p}, p}$ by [90, 2.4.2(16)].

On $a$ and $f$ we assume the following conditions:

(ii) Assume each $a_{\alpha}: J_{0} \times \mathbb{R}^{d} \times \mathbb{R} \times \mathbb{R}^{d} \rightarrow \mathbb{C}$ is a measurable function such that $\sup _{t, x, y, z}\left|a_{\alpha}(t, x, y, z)\right|<\infty$ and there is an $\theta \in(0, \pi)$ and $\kappa \in(0,1)$ such that for all $t \in J_{0}, x, z \in \mathbb{R}^{d}, y \in \mathbb{R}$,

$$
\sum_{|\alpha|=2} a_{\alpha}(t, x, y, z) \xi^{\alpha} \subset \Sigma_{\theta} \text { and }\left|\sum_{|\alpha|=2} a_{\alpha}(t, x, y, z) \xi^{\alpha}\right| \geq \kappa, \quad \xi \in \mathbb{R}^{d},|\xi|=1 .
$$

(iii) Assume that for every $R>0$ there exists a function $\omega_{R}: \mathbb{R}_{+} \rightarrow \mathbb{R}_{+}$with $\lim _{\varepsilon \downarrow 0} \omega_{R}(\varepsilon)=0$ such that for all $t \in J_{0}, x_{1}, x_{2} \in \mathbb{R}^{d},|y|,|z| \leq R$,

$$
\left|a_{\alpha}\left(t, x_{1}, y, z\right)-a_{\alpha}\left(t, x_{2}, y, z\right)\right| \leq \omega_{R}\left(\left|x_{1}-x_{2}\right|\right) .
$$

(iv) Assume that for each $|\alpha|=2$ for every $R>0$ there exists a constant $C_{\alpha}(R)$ such that for all $t \in J_{0}, x \in \mathbb{R}^{d},\left|y_{1}\right|,\left|y_{2}\right| \leq R$, and $\left|z_{1}\right|,\left|z_{2}\right| \leq R$,

$$
\left|a_{\alpha}\left(t, x, y_{1}, z_{1}\right)-a_{\alpha}\left(t, x, y_{2}, z_{2}\right)\right| \leq C_{\alpha}(R)\left(\left|y_{1}-y_{2}\right|+\left|z_{1}-z_{2}\right|\right),
$$

(v) Assume $f: J_{0} \times \mathbb{R}^{d} \times \mathbb{R} \times \mathbb{R}^{d} \rightarrow \mathbb{C}$ is a measurable function such that

$$
\int_{J_{0}}\left(\int_{\mathbb{R}^{d}}|f(t, x, 0,0)|^{q} d x\right)^{\frac{p}{q}} d t<\infty .
$$

For every $R>0$ there exists a function $\phi(R) \in L^{p}\left(J_{0}\right)$ such that for all $t \in J_{0}$, $x \in \mathbb{R}^{d},\left|y_{1}\right|,\left|y_{2}\right| \leq R$ and $\left|z_{1}\right|,\left|z_{2}\right| \leq R$,

$$
\left|f\left(t, x, y_{1}, z_{1}\right)-f\left(t, x, y_{2}, z_{2}\right)\right|_{X_{0}} \leq \phi(R)(t)\left(\left|y_{1}-y_{2}\right|+\left|z_{1}-z_{2}\right|\right) .
$$

Let $\operatorname{MR}^{p}(J)=W^{1, p}\left(J ; L^{q}\left(\mathbb{R}^{d}\right)\right) \cap L^{p}\left(J ; W^{2, q}\left(\mathbb{R}^{d}\right)\right)$ and note that by Eq. 6.5

$$
\operatorname{MR}^{p}(J) \hookrightarrow C\left(J ; X_{p}\right) \hookrightarrow C\left(J ; C^{1+\delta}\left(\mathbb{R}^{d}\right)\right) .
$$

In order to apply Theorem 6.1 to obtain local well-posedness define $A: J_{0} \times X_{p} \rightarrow$ $\mathscr{L}\left(X_{1}, X_{0}\right)$ and $F: J_{0} \times X_{p} \rightarrow X_{0}$ by

$$
\begin{aligned}
(A(t, v) u)(x) & =\sum_{|\alpha| \leq 2} a_{\alpha}(t, x, v(x), \nabla v(x)) D^{\alpha} u(x), \\
F(t, u)(x) & =f(t, x, u(x), \nabla u(x)) .
\end{aligned}
$$


Then $A$ and $F$ satisfy the conditions of Theorem 6.1. Indeed, applying Eq. 6.6 we find that for $R>0$ and $\left\|v_{1}\right\|_{X_{p}},\left\|v_{2}\right\|_{X_{p}} \leq R$ and $u \in X_{1}$,

$$
\begin{aligned}
\left\|A\left(t, v_{1}\right) u-A\left(t, v_{2}\right) u\right\|_{X_{0}} & \leq K(R)\left(\left\|v_{1}-v_{2}\right\|_{X_{0}}+\left\|\nabla v_{1}-\nabla v_{2}\right\|_{X_{0}}\right)\|u\|_{X_{1}} \\
& \leq K(R) C\left\|v_{1}-v_{2}\right\|_{X_{p}}\|u\|_{X_{1}} .
\end{aligned}
$$

Here we have used that for $k \in\{0,1\}$ and $v \in X_{p},\left\|D^{k} v\right\|_{\infty} \leq C\|v\|_{X_{p}}$ by Eq. 6.5.

Next we check that for every $g \in X_{p}, A(\cdot, g)$ has maximal $L^{p}$-regularity. In order to do so we check that $A(t, g)$ satisfies the conditions of Theorem 5.4. Indeed, let $R=$ $\|g\|_{C^{1}\left(\mathbb{R}^{d}\right)}<\infty$. By Eq. 6.5, $g \in C^{1+\delta}\left(\mathbb{R}^{d}\right)$ and therefore,

$$
\begin{aligned}
& \left|a_{\alpha}\left(t, x_{1}, g\left(x_{1}\right), \nabla g\left(x_{1}\right)\right)-a_{\alpha}\left(t, x_{2}, g\left(x_{2}\right), \nabla g\left(x_{2}\right)\right)\right| \\
& \quad \leq\left|a_{\alpha}\left(t, x_{1}, g\left(x_{1}\right), \nabla g\left(x_{1}\right)\right)-a_{\alpha}\left(t, x_{2}, g\left(x_{1}\right), \nabla g\left(x_{1}\right)\right)\right| \\
& \quad+\left|a_{\alpha}\left(t, x_{2}, g\left(x_{1}\right), \nabla g\left(x_{1}\right)\right)-a_{\alpha}\left(t, x_{2}, g\left(x_{2}\right), \nabla g\left(x_{2}\right)\right)\right| \\
& \quad \leq \omega_{R}\left(\left|x_{1}-x_{2}\right|\right)+\left|g\left(x_{1}\right)-g\left(x_{2}\right)\right|+\left|\nabla g\left(x_{1}\right)-\nabla g\left(x_{2}\right)\right| \\
& \quad \leq \omega_{R}\left(\left|x_{1}-x_{2}\right|\right)+\|g\|_{C^{1+\delta}\left(\mathbb{R}^{d}\right)}\left(\left|x_{1}-x_{2}\right|+\left|x_{1}-x_{2}\right|^{\delta}\right) .
\end{aligned}
$$

Thus $A(t, g)$ satisfies the required continuity condition in the space variable. Hence Theorem 5.4 yields that $A\left(\cdot, u_{0}\right)$ has maximal $L^{p}$-regularity. The conditions on $F$ can be checked in a similar way and we obtain the following result as a consequence of Theorem 6.1.

Theorem 6.2 Assume the above conditions on $p, q \in(1, \infty)$ and $a_{\alpha}$ and $f$. Let $g \in X_{p}:=$ $B_{q, p}^{2\left(1-\frac{1}{p}\right)}\left(\mathbb{R}^{d}\right)$ be arbitrary. Then there is a $T \in\left(0, T_{0}\right]$ and radius $\varepsilon>0$ both depending on $g$ such that for all $u_{0} \in B_{\varepsilon}=\left\{v \in X_{p}:\|v-g\|_{X_{p}} \leq \varepsilon\right\}$, Eq. 6.3 admits a unique solution

$$
u \in W^{1, p}\left(J ; L^{q}\left(\mathbb{R}^{d}\right)\right) \cap L^{p}\left(J ; W^{2, q}\left(\mathbb{R}^{d}\right)\right) \cap C\left(J ; X_{p}\right) .
$$

Moreover, there is a constant $C$ such that for all $u_{0}, v_{0} \in B_{\varepsilon}$ the corresponding solutions $u$ and $v$ satisfy

$$
\|u-v\|_{W^{1, p}\left(J ; L^{q}\left(\mathbb{R}^{d}\right)\right)}+\|u-v\|_{L^{p}\left(J ; W^{2, q}\left(\mathbb{R}^{d}\right)\right)}+\|u-v\|_{C\left(J ; X_{p}\right)} \leq C\|x-y\|_{X_{p}} .
$$

\section{Remark 6.3}

(1) If $a_{\alpha}$ only depends on $u$ and not on its derivatives, then one can replace Eq. 6.4 by the condition $2\left(1-\frac{1}{p}\right)-\frac{d}{q}>0$.

(2) Theorem 6.2 can be extended to higher order equations. Then $a_{\alpha}$ is allowed to depend on the $(m-1)$-th derivatives of $u$. Moreover, by [33] one can also consider higher order systems.

\subsection{Proof of Theorem 6.1}

From the trace estimate (4.18) and a simple reflection argument one sees see that there exists a constant $C$ independent of $T$ such that for all $u \in \operatorname{MR}^{p}(J)$ with $u(0)=0$ one has

$$
\|u\|_{C\left([0, T] ; X_{p}\right)} \leq C_{\operatorname{Tr}}\|u\|_{\mathrm{MR}^{p}(J)} .
$$

Without the assumption $u(0)=0$, one still has the above estimate but with a constant which blows up as $T \downarrow 0$ (see Eq. 4.17 and use a translation argument). 
For $u, v \in \operatorname{MR}(J)$ with $u(0)=v(0) \in X_{p}$, the following consequence of Eq. 6.7 will be used frequently:

$$
\begin{aligned}
\|u\|_{C\left(J ; X_{p}\right)} & \leq\|u-v\|_{C\left(J ; X_{p}\right)}+\|v\|_{C\left(J ; X_{p}\right)} \\
& \leq C_{\operatorname{Tr}}\|u-v\|_{\mathrm{MR}^{p}((0, T))}+\|v\|_{C\left(J ; X_{p}\right)} .
\end{aligned}
$$

Proof of Theorem 6.1 We modify the presentation in [59] to our setting. By the assumption and Proposition 4.15 we know that for each $x \in X_{p}$, there exists a unique solution $w^{x} \in$ $\operatorname{MR}^{p}(J)$ of the problem

$$
\left\{\begin{array}{l}
w^{\prime}(t)+A\left(t, x_{0}\right) w(t)=F\left(t, x_{0}\right), t \in J_{0} \\
w(0)=x
\end{array}\right.
$$

Moreover, by linearity

$$
\left\|w^{x}-w^{y}\right\|_{\mathrm{MR}^{p}\left(J_{0}\right)} \leq C_{0}\|x-y\|_{X_{p}} .
$$

By Eq. 4.17 and a translation argument we see that

$$
\left\|w^{x}-w^{y}\right\|_{C\left(J_{0} ; X_{p}\right)} \leq C_{1}\left\|w^{x}-w^{y}\right\|_{\mathrm{MR}^{p}\left(J_{0}\right)} \leq C_{1} C_{2}\|x-y\|_{X_{p}} .
$$

Step 1. Let $C_{A}$ be the maximal $L^{p}$-regularity constant of $A\left(\cdot, u_{0}\right)$. We show that for a certain set of function $u \in \mathrm{MR}^{p}(J)$ maximal $L^{p}$-regularity holds with constant $2 C_{A}$. Fix $R>0$. Since $w^{x_{0}}:[0, T] \rightarrow X_{p}$ is continuous we can find $T \in\left(0, T_{0}\right]$ such that

$$
\left\|w^{x_{0}}(t)-x_{0}\right\|_{X_{p}} \leq \frac{1}{4 C(R) C_{A}}, \quad t \in[0, T] .
$$

Let

$$
r_{0}:=\frac{1}{4 C(R) C_{A}\left(C_{\mathrm{Tr}}+C_{\mathrm{Tr}} C_{2}+C_{1} C_{2}\right)}
$$

and write

$$
\mathbb{B}_{r_{0}}=\left\{v \in \operatorname{MR}^{p}(J):\left\|v(0)-x_{0}\right\|_{X_{p}} \leq r_{0} \text { and }\left\|v-w^{x_{0}}\right\|_{\mathrm{MR}^{p}(J)} \leq r_{0}\right\} .
$$

From the assumptions we see that for all $v \in \mathbb{B}_{r_{0}}$ and $t \in[0, T]$, writing $x=v(0)$,

$$
\begin{aligned}
& \left\|v(t)-x_{0}\right\|_{X_{p}} \\
& \leq\left\|v(t)-w^{x}(t)\right\|_{X_{p}}+\left\|w^{x}(t)-w^{x_{0}}(t)\right\|_{X_{p}}+\left\|w^{x_{0}}(t)-x_{0}\right\|_{X_{p}} \\
& \leq C_{\operatorname{Tr}}\left\|v-w^{x}\right\|_{\mathrm{MR}^{p}(J)}+C_{1} C_{2}\left\|x-x_{0}\right\|_{X_{p}}+\frac{1}{4 C(R) C_{A}} \\
& \leq C_{\operatorname{Tr}} r_{0}+C_{\operatorname{Tr}}\left\|w^{x_{0}}-w^{x}\right\|_{\mathrm{MR}^{p}(J)}+C_{1} C_{2}\left\|x-x_{0}\right\|_{X_{p}}+\frac{1}{4 C(R) C_{A}} \\
& \leq C_{\operatorname{Tr}} r_{0}+C_{\operatorname{Tr}} C_{2} r_{0}+C_{1} C_{2} r_{0}+\frac{1}{4 C(R) C_{A}} \leq \frac{1}{2 C(R) C_{A}},
\end{aligned}
$$

where we used Eqs. 6.7 and 6.9. Therefore by Eq. 6.11 and the assumption

$$
\left\|A(t, v(t))-A\left(t, x_{0}\right)\right\|_{\mathscr{L}\left(X_{1}, X_{0}\right)} \leq C(R)\left\|v(t)-x_{0}\right\|_{X_{p}} \leq \frac{1}{2 C_{A}} .
$$

Now Proposition 4.18 yields that $A(\cdot, v(\cdot))$ has maximal $L^{p}$-regularity with constant $2 C_{A}$ for each $v \in \mathbb{B}_{r_{0}}$. 
Step 2. Let $R=1+C_{\operatorname{Tr}}+C_{\operatorname{Tr}} C_{2}+C_{1} C_{2}+\left\|w^{x_{0}}\right\|_{C\left(J_{0} ; X_{p}\right)}$. Fix $0<r \leq \min \left\{1, r_{0}\right\}$ and $T$ as in Step 1. Note that by Eqs. 6.8 and 6.9 for $v \in \mathbb{B}_{r}$ and $x=v(0)$,

$$
\begin{aligned}
& \|v\|_{C\left(J ; X_{p}\right)} \leq C_{\operatorname{Tr}}\left\|v-w^{x}\right\|_{\mathrm{MR}^{p}(J)}+\left\|w^{x}-w^{x_{0}}\right\|_{C\left(J ; X_{p}\right)}+\left\|w^{x_{0}}\right\|_{C\left(J ; X_{p}\right)} \\
& \leq C_{\operatorname{Tr}}\left\|v-w^{x_{0}}\right\|_{\mathrm{MR}^{p}(J)}+C_{\operatorname{Tr}}\left\|w^{x_{0}}-w^{x}\right\|_{\mathrm{MR}^{p}(J)}+C_{1} C_{2}\left\|x-x_{0}\right\|_{X_{p}}+\left\|w^{x_{0}}\right\|_{C\left(J ; X_{p}\right)} \\
& \leq C_{\operatorname{Tr}} r+\left(C_{\operatorname{Tr}} C_{2}+C_{1} C_{2}\right)\left\|x-x_{0}\right\|_{X_{p}}+\left\|w^{x_{0}}\right\|_{C\left(J ; X_{p}\right)} \\
& \leq C_{\operatorname{Tr}} r+\left(C_{\operatorname{Tr}} C_{2}+C_{1} C_{2}\right) r+\left\|w^{x_{0}}\right\|_{C\left(J ; X_{p}\right)} \leq R,
\end{aligned}
$$

where we used $r \leq 1$. Similarly, for $x \in B_{r},\|x\|_{X_{p}} \leq r+\left\|x_{0}\right\|_{X_{p}} \leq R$.

For $x \in B_{r}$, let $\mathbb{B}_{r, x} \subseteq \mathbb{B}_{r}$ be defined by

$$
\mathbb{B}_{r, x}=\left\{u \in \operatorname{MR}^{p}(J): u(0)=x \text { and }\left\|u-w^{x_{0}}\right\|_{\mathrm{MR}^{p}(J)} \leq r\right\} .
$$

Before we introduce a fixed point operator argument on $\mathbb{B}_{r, x}$, let

$$
\begin{aligned}
f\left(v_{1}, v_{2}\right) & =F\left(t, v_{1}(t)\right)-F\left(t, v_{2}(t)\right), \\
a\left(v_{1}, v_{2}, v_{3}\right)(t) & =\left(A\left(t, v_{2}(t)\right)-A\left(t, v_{1}(t)\right)\right) v_{3}(t) .
\end{aligned}
$$

for $v_{j} \in \mathbb{B}_{r, x_{j}}$ with $x_{j} \in B_{r}$ for $j \in\{1,2\}$ and $v_{3} \in \operatorname{MR}^{p}(J)$. Observe that by Eqs. 6.8 and 6.9

$$
\begin{aligned}
\left\|v_{1}-v_{2}\right\|_{C\left(J ; X_{p}\right)} & \leq C_{\operatorname{Tr}}\left\|v_{1}-v_{2}-\left(w^{x_{1}}-w^{x_{2}}\right)\right\|_{\mathrm{MR}^{p}(J)}+\left\|w^{x_{1}}-w^{x_{2}}\right\|_{C\left(J ; X_{p}\right)} \\
& \leq C_{\operatorname{Tr}}\left\|v_{1}-v_{2}\right\|_{\mathrm{MR}^{p}(J)}+\left(C_{\operatorname{Tr}} C_{2}+C_{1} C_{2}\right)\left\|x_{1}-x_{2}\right\|_{X_{p}} .
\end{aligned}
$$

Let $C_{J}=\left\|\phi_{R}\right\|_{L^{p}(J)}$. For $f$ we find

$$
\begin{aligned}
\left\|f\left(v_{1}, v_{2}\right)\right\|_{L^{p}\left(J ; X_{0}\right)} & \leq\left\|\phi_{R}\left(v_{1}-v_{2}\right)\right\|_{L^{p}\left(J ; X_{p}\right)} \leq C_{J}\left\|v_{1}-v_{2}\right\|_{C\left(J ; X_{p}\right)} \\
& \leq C_{J} C_{\operatorname{Tr}}\left\|v_{1}-v_{2}\right\|_{\mathrm{MR}^{p}(J)}+C_{J} C_{3}\left\|x_{1}-x_{2}\right\|_{X_{p}},
\end{aligned}
$$

where $C_{3}=\left(C_{\mathrm{Tr}} C_{2}+C_{1} C_{2}\right)$. Similarly, applying the estimate for $v_{1}-v_{2}$ again,

$$
\begin{aligned}
\left\|a\left(v_{1}, v_{2}, v_{3}\right)\right\|_{L^{p}\left(J ; X_{0}\right)} & \leq C(R)\|\| v_{2}-v_{1}\left\|_{X_{p}}\right\| v_{3}\left\|_{X_{1}}\right\| \|_{L^{p}\left(J ; X_{0}\right)} \\
& \leq C(R)\left\|v_{1}-v_{2}\right\|_{C\left(J ; X_{p}\right)}\left\|v_{3}\right\|_{\mathrm{MR}^{p}(J)} \\
& \leq C(R)\left\|v_{3}\right\|_{\mathrm{MR}^{p}(J)}\left[C_{\operatorname{Tr}}\left\|v_{1}-v_{2}\right\|_{\mathrm{MR}^{p}(J)}+C_{3}\left\|x_{1}-x_{2}\right\|_{X_{p}}\right] .
\end{aligned}
$$

For $v \in \mathbb{B}_{r, x}$ and $x \in B_{r}$ let $L_{x}(v)=u \in \operatorname{MR}^{p}(J)$ denote the solution of

$$
\left\{\begin{array}{l}
u^{\prime}(t)+A(t, v(t)) u(t)=F(t, v(t)), t \in J_{0} \\
u(0)=x .
\end{array}\right.
$$

For $v_{1}, v_{2}$ as before let $u_{j}:=L_{x_{j}}\left(v_{j}\right)$ for $j \in\{1,2\}$. We find that $u:=u_{1}-u_{2}$ in $\operatorname{MR}^{p}(J)$ satisfies $u(0)=x_{1}-x_{2}$ and

$$
u^{\prime}(t)+A\left(t, v_{1}(t)\right) u(t)=f\left(v_{1}, v_{2}\right)(t)+a\left(v_{1}, v_{2}, u_{2}\right)(t), \quad t \in J .
$$

Therefore, by Step 1, Proposition 4.15 and the previous estimates, we find

$$
\begin{aligned}
& \left\|L_{x_{1}}\left(v_{1}\right)-L_{x_{2}}\left(v_{2}\right)\right\|_{\mathrm{MR}^{p}(J)} \\
& \quad \leq 2 C_{A}\left(\left\|x_{1}-x_{2}\right\|_{X_{p}}+\left\|f\left(v_{1}, v_{2}\right)\right\|_{L^{p}\left(J ; X_{0}\right)}+\left\|a\left(v_{1}, v_{2}, u_{2}\right)\right\|_{L^{p}\left(J ; X_{0}\right)}\right) \\
& \leq K_{1}\left(\left\|u_{2}\right\|_{\mathrm{MR}^{p}(J)}\right)\left\|x_{1}-x_{2}\right\|_{X_{p}}+K_{2}\left(\left\|u_{2}\right\|_{\mathrm{MR}^{p}(J)}\right)\left\|v_{1}-v_{2}\right\|_{\mathrm{MR}^{p}(J)},
\end{aligned}
$$

where for $s \geq 0$,

$$
\begin{aligned}
& K_{1}(s)=2 C_{A}\left(1+C_{J} C_{3}+C(R) C_{3} s\right), \\
& K_{2}(s)=2 C_{A}\left[C_{J} C_{\operatorname{Tr}}+C(R) C_{\operatorname{Tr}} s\right] .
\end{aligned}
$$


Extending the definitions of $L, f$ and $a$ in the obvious way we can write $w^{x_{0}}=L_{x_{0}}\left(x_{0}\right)$. Estimating as before, one sees that for $x \in B_{r}$ and $v \in \mathbb{B}_{r, x}$,

$$
\begin{aligned}
& \left\|L_{x}(v)-L_{x_{0}}\left(x_{0}\right)\right\|_{\mathrm{MR}^{p}(J)} \\
& \leq 2 C_{A}\left(\left\|x-x_{0}\right\|_{X_{p}}+\| f\left(v, x_{0}\left\|_{L^{p}\left(J ; X_{0}\right)}+\right\| a\left(v, x_{0}, w^{x_{0}}\right) \|_{L^{p}\left(J ; X_{0}\right)}\right)\right. \\
& \leq 2 C_{A}\left(\left\|x-x_{0}\right\|_{X_{p}}+\left[C_{J}+C(R)\left\|w^{x_{0}}\right\|_{\mathrm{MR}^{p}(J)}\right]\left\|v-x_{0}\right\|_{C\left(J ; X_{p}\right)}\right), \\
& \leq 2 C_{A}\left(\left\|x-x_{0}\right\|_{X_{p}}+\left[C_{J}+C(R)\left\|w^{x_{0}}\right\|_{\mathrm{MR}^{p}(J)}\right] \frac{1}{2 C(R) C_{A}}\right),
\end{aligned}
$$

where in the last step we used Eq. 6.11.

Choose $0<r \leq \min \left\{1, r_{0}\right\}$ such that

$$
4 C_{A} r C(R) C_{\operatorname{Tr}} \leq \frac{1}{4}
$$

Choose $T$ such that Eq. 6.10 holds,

$$
2 C_{A} C_{J} C_{\operatorname{Tr}} \leq \frac{1}{4}, \quad \frac{C_{J}}{C(R)} \leq \frac{r}{4}, \text { and }\left\|w^{x_{0}}\right\|_{\mathrm{MR}^{p}(J)} \leq \frac{r}{4} .
$$

Let $\varepsilon=\min \left\{\frac{r}{4 C_{A}}, r\right\}$. Then from Eq. 6.13 we obtain that for all $x \in B_{\varepsilon}, L_{x}$ maps $\mathbb{B}_{r, x}$ into itself. In particular, for all $x \in B_{\varepsilon}$ and $v \in \mathbb{B}_{r, x}$,

$$
\left\|L_{x}(v)\right\|_{\mathrm{MR}^{p}(J)} \leq\left\|L_{x}(v)-w^{x_{0}}\right\|_{\mathrm{MR}^{p}(J)}+\left\|w^{x_{0}}\right\|_{\mathrm{MR}^{p}(J)} \leq r+\frac{r}{4} \leq 2 r .
$$

Moreover, for all $x_{j} \in B_{\varepsilon}$ and $v_{j} \in \mathbb{B}_{r, x_{j}}$ for $j \in\{1,2\}$,

$$
\left\|L_{x_{1}}\left(v_{1}\right)-L_{x_{2}}\left(v_{2}\right)\right\|_{\mathrm{MR}^{p}(J)} \leq K_{1}(2)\left\|x_{1}-x_{2}\right\|_{X_{p}}+\frac{1}{2}\left\|v_{1}-v_{2}\right\|_{\mathrm{MR}^{p}(J)},
$$

where we used Eqs. 6.12 and 6.14. In particular, $L_{x}$ defines a contraction on $\mathbb{B}_{r, x}$ and by the Banach contraction principle we find that there exists a unique $u \in \mathbb{B}_{r, x}$ such that $L_{x}(u)=u$. This yields the required result.

The final estimate of the theorem follows from Eq. 6.15.

Acknowledgments The authors thank Doyoon Kim for pointing out the references [58, 60]. We also thank Nick Lindemulder and Jan Rozendaal for careful reading and useful comments. Finally, we thank the referee for his/her kind suggestions to improve the paper.

Open Access This article is distributed under the terms of the Creative Commons Attribution 4.0 International License (http://creativecommons.org/licenses/by/4.0/), which permits unrestricted use, distribution, and reproduction in any medium, provided you give appropriate credit to the original author(s) and the source, provide a link to the Creative Commons license, and indicate if changes were made.

\section{References}

1. Abels, H., Terasawa, Y.: On Stokes operators with variable viscosity in bounded and unbounded domains. Math. Ann. 344(2), 381-429 (2009)

2. Acquistapace, P., Terreni, B.: A unified approach to abstract linear nonautonomous parabolic equations. Rend. Sem. Mat. Univ. Padova 78, 47-107 (1987)

3. Albrecht, D., Duong, X.T., McIntosh, A.: Operator theory and harmonic analysis. In: Instructional Workshop on Analysis and Geometry, Part III (Canberra, 1995), Volume 34 of Proc. Centre Math. Appl. Austral. Nat. Univ., pp. 77-136. Canberra, Austral. Nat. Univ. (1996) 
4. Amann, H.: Linear and Quasilinear Parabolic Problems. Vol. I, Abstract Linear Theory, Volume 89 of Monographs in Mathematics. Birkhäuser Boston Inc., Boston (1995)

5. Amann, H.: Maximal regularity for nonautonomous evolution equations. Adv. Nonlinear Stud. 4(4), 417-430 (2004)

6. Amann, H.: Maximal regularity and quasilinear parabolic boundary value problems. In: Recent Advances in Elliptic and Parabolic Problems, pp. 1-17. World Sci. Publ., Hackensack (2005)

7. Arendt, W., Chill, R., Fornaro, S., Poupaud, C.: $L^{p}$-maximal regularity for non-autonomous evolution equations. J. Differ. Equ. 237(1), 1-26 (2007)

8. Arendt, W., Dier, D., Laasri, H., Ouhabaz, E.M.: Maximal regularity for evolution equations governed by non-autonomous forms. Adv. Differential Equations 19(11-12), 1043-1066 (2014)

9. Auscher, P., McIntosh, A., Nahmod, A.: Holomorphic functional calculi of operators, quadratic estimates and interpolation. Indiana Univ. Math. J. 46(2), 375-403 (1997)

10. Bastero, J., Milman, M., Ruiz, F.J.: On the connection between weighted norm inequalities, commutators and real interpolation. Mem. Amer. Math. Soc. 154(731), viii+80 (2001)

11. Bergh, J., Löfström, J.: Interpolation Spaces. An Introduction. Springer, Berlin (1976). Grundlehren der Mathematischen Wissenschaften, No. 223

12. Brudnyı̆, Y.A., Krugljak, N.Y.: Interpolation functors and interpolation spaces. Vol. I, volume 47 of North-Holland Mathematical Library. North-Holland Publishing Co., Amsterdam (1991). Translated from the Russian by Natalie Wadhwa, With a preface by Jaak Peetre

13. Chill, R., Fiorenza, A.: Singular integral operators with operator-valued kernels, and extrapolation of maximal regularity into rearrangement invariant Banach function spaces. J. Evol. Equ., 1-34 (2014)

14. Clément, P., Li, S.: Abstract parabolic quasilinear equations and application to a groundwater flow problem. Adv. Math. Sci. Appl. 3(Special Issue), 17-32 (Unknown Month 1993)

15. Clément, P., de Pagter, B., Sukochev, F.A., Witvliet, H.: Schauder decompositions and multiplier theorems. Studia Math. 138(2), 135-163 (2000)

16. Clément, P., Prüss, J.: Global existence for a semilinear parabolic Volterra equation. Math. Z. 209(1), 17-26 (1992)

17. Cowling, M., Doust, I., McIntosh, A., Yagi, A.: Banach space operators with a bounded $h^{\infty}$ functional calculus. J. Austral. Math. Soc. Ser. A 60(1), 51-89 (1996)

18. Cruz-Uribe, D.V., Martell, J.M., Pérez, C.: Weights, Extrapolation and the Theory of Rubio De Francia, Volume 215 of Operator Theory: Advances and Applications. Birkhäuser/Springer Basel AG, Basel (2011)

19. David, G., Journé, J.-L.: A boundedness criterion for generalized calderón-Zygmund operators. Ann. of Math. (2) 120(2), 371-397 (1984)

20. David, G., Journé, J.-L., Semmes, S.: Opérateurs de calderón-Zygmund, fonctions para-accrétives et interpolation. Rev. Mat. Iberoamericana 1(4), 1-56 (1985)

21. Denk, R., Geissert, M., Hieber, M., Saal, J., Sawada, O.: The spin-coating process: analysis of the free boundary value problem. Comm. Partial Differential Equations 36(7), 1145-1192 (2011)

22. Denk, R., Hieber, M., Prüss, J.: R-boundedness, fourier multipliers and problems of elliptic and parabolic type. Mem. Amer. Math. Soc. 166(788) (2003)

23. Dier, D.: Non-autonomous maximal regularity for forms of bounded variation. J. Math. Anal. Appl 425(1), 33-54 (2015)

24. Dier, D., Zacher, R.: Non-autonomous maximal regularity in Hilbert spaces. Online first in J. Evol Equ. (2016)

25. Diestel, J., Jarchow, H., Tonge, A.: Absolutely Summing Operators, Volume 43 of Cambridge Studies in Advanced Mathematics. Cambridge University Press, Cambridge (1995)

26. Dong, H., Kim, D.: On the $L_{p}$-solvability of higher order parabolic and elliptic systems with BMO coefficients. Arch. Ration. Mech. Anal. 199(3), 889-941 (2011)

27. Dore, G.: Maximal regularity in $L^{p}$ spaces for an abstract Cauchy problem. Adv. Differential Equations 5(1-3), 293-322 (2000)

28. Engel, K.-J., Nagel, R.: One-Parameter Semigroups for Linear Evolution Equations, Volume 194 of Graduate Texts in Mathematics. Springer, New York (2000)

29. Fackler, S.: The Kalton-Lancien theorem revisited: maximal regularity does not extrapolate. J. Funct. Anal. 266(1), 121-138 (2014)

30. Fackler, S.: J.-L. Lions' problem concerning maximal regularity of equations governed by nonautonomous forms. To appear in Ann. Inst. H. Poincaré Anal Non linéaire (2016)

31. Fröhlich, A.: The Stokes operator in weighted $L^{q}$-spaces. II. Weighted resolvent estimates and maximal $L^{p}$-regularity. Math. Ann. 339(2), 287-316 (2007)

32. Gallarati, C., Lorist, E., Veraar, M.C.: On the $\ell^{s}$-boundedness of a family of integral operators. to appear in Revista Matemàtica Iberoamericana. arXiv:1410.6657 (2015) 
33. Gallarati, C., Veraar, M.C.: Evolution families and maximal regularity for systems of parabolic equations. to appear in Advances in Differential Equations. See arxiv preprint server, arXiv:1510.07643

34. García-Cuerva, J., Rubio de Francia, J.L.: Weighted norm inequalities and related topics, volume 116 of North-Holland Mathematics Studies. North-Holland Publishing Co., Amsterdam (1985). Notas de Matemática [Mathematical Notes], 104

35. Geissert, M., Hess, M., Hieber, M., Schwarz, C., Stavrakidis, K.: Maximal $L^{p}-L^{q}$-estimates for the Stokes equation: a short proof of Solonnikov's theorem. J. Math. Fluid Mech. 12(1), 47-60 (2010)

36. Gilbarg, D., Trudinger, N.S.: Elliptic Partial Differential Equations of Second Order. Classics in Mathematics. Springer, Berlin (2001). Reprint of the 1998 edition

37. Grafakos, L.: Classical Fourier Analysis, Volume 249 of Graduate Texts in Mathematics, 2nd edn. Springer, New York (2008)

38. Grafakos, L.: Modern Fourier Analysis, Volume 250 of Graduate Texts in Mathematics, 2nd edn. Springer, New York (2009)

39. Grisvard, P.: Espaces intermédiaires entre espaces de Sobolev avec poids. Ann. Scuola Norm. Sup. Pisa (3) 17, 255-296 (1963)

40. Haak, B.H., Haase, M.: Square function estimates and functional calculi. arXiv:1311.0453 (2013)

41. Haak, B.H., Ouhabaz, E.M.: Maximal regularity for non-autonomous evolution equations. Math. Ann. 363(3-4), 1117-1145 (2015)

42. Haase, M.H.A.: The Functional Calculus for Sectorial Operators, Volume 169 of Operator Theory: Advances and Applications. Basel, Birkhäuser (2006)

43. Haller, R., Heck, H., Hieber, M.: Muckenhoupt weights and maximal $L^{p}$-regularity. Arch. Math. (Basel) 81(4), 422-430 (2003)

44. Haller-Dintelmann, R., Rehberg, J.: Maximal parabolic regularity for divergence operators including mixed boundary conditions. J. Differ. Equ. 247(5), 1354-1396 (2009)

45. Hänninen, T.S., Hytönen, T.P.: The $A_{2}$ theorem and the local oscillation decomposition for Banach space valued functions. J. Operator Theory 72(1), 193-218 (2014)

46. Hardy, G.H., Littlewood, J.E., Pólya, G.: Inequalities. Cambridge Mathematical Library. Cambridge University Press, Cambridge (1988). Reprint of the 1952 edition

47. Hytönen, T.P.: An operator-valued $T b$ theorem. J. Funct. Anal. 234(2), 420-463 (2006)

48. Hytönen, T.P.: The vector-valued nonhomogeneous Tb theorem. Int. Math. Res. Not. IMRN 2, 451-511 (2014)

49. Hytönen, T.P., Weis, L.W.: A $T 1$ theorem for integral transformations with operator-valued kernel. J. Reine Angew. Math. 599, 155-200 (2006)

50. Hytönen, T.P., Weis, L.W.: Singular convolution integrals with operator-valued kernel. Math. Z. 255(2), 393-425 (2007)

51. Kaljabin, G.A.: Generalized method of traces in the theory of the interpolation of Banach spaces. Mat. Sb. (N.S.), 106(148)(1), 85-93, 144 (1978)

52. Kalton, N.J., Kucherenko, T.: Operators with an absolute functional calculus. Math. Ann. 346(2), 259$306(2010)$

53. Kalton, N.J., Lancien, G.: A solution to the problem of $L^{p}$-maximal regularity. Math. Z. 235(3), 559-568 (2000)

54. Kalton, N.J., Lancien, G.: $L^{p}$-maximal regularity on Banach spaces with a Schauder basis. Arch. Math. (Basel) 78(5), 397-408 (2002)

55. Kalton, N.J., Weis, L., The $H^{\infty}$-calculus and square function estimates (2014). arXiv: 1411.0472

56. Kalton, N.J., Weis, L.W.: The $H^{\infty}$-calculus and sums of closed operators. Math. Ann. 321(2), 319-345 (2001)

57. Kim, D.: Elliptic and parabolic equations with measurable coefficients in $L_{p}$-spaces with mixed norms. Methods Appl. Anal. 15(4), 437-467 (2008)

58. Kim, D.: Parabolic equations with partially BMO coefficients and boundary value problems in Sobolev spaces with mixed norms. Potential Anal. 33(1), 17-46 (2010)

59. Köhne, M., Prüss, J., Wilke, M.: On quasilinear parabolic evolution equations in weighted $L_{p}$-spaces. J. Evol. Equ. 10(2), 443-463 (2010)

60. Krylov, N.V.: The heat equation in $L_{q}\left((0, T), L_{p}\right)$-spaces with weights. SIAM J. Math. Anal. 32(5), $1117-1141$ (2001)

61. Krylov, N.V.: Lectures on Elliptic and Parabolic Equations in Sobolev Spaces, Volume 96 of Graduate Studies in Mathematics. American Mathematical Society, Providence (2008)

62. Kunstmann, P.C., Weis, L.: Maximal $L_{p}$-regularity for parabolic equations, Fourier multiplier theorems and $H^{\infty}$-functional calculus. In: Functional Analytic Methods for Evolution Equations, Volume 1855 of Lecture Notes in Math, pp. 65-311. Springer, Berlin (2004) 
63. Latushkin, Y., Prüss, J., Schnaubelt, R.: Center manifolds and dynamics near equilibria of quasilinear parabolic systems with fully nonlinear boundary conditions. Discrete Contin. Dyn. Syst. Ser. B 9(3-4), 595-633 (2008)

64. Le Merdy, C.: On square functions associated to sectorial operators. Bull. Soc. Math. France 132(1), 137-156 (2004)

65. Lions, J.-L.: Équations différentielles opérationnelles et problèmes aux limites, Die Grundlehren Der Mathematischen Wissenschaften Bd. 111. Springer, Berlin (1961)

66. Lions, J.-L.: Contrôle optimal de systèmes gouvernés par des équations aux dérivées partielles. Avant Propos de P. Lelong, Dunod, Paris (1968)

67. Lunardi, A.: Analytic Semigroups and Optimal Regularity in Parabolic Problems Progress in Nonlinear Differential Equations and their Applications, vol. 16. Basel, Birkhäuser (1995)

68. McIntosh, A.: Operators Which have an $H_{\infty}$ Functional Calculus. In: Miniconference on Operator Theory and Partial Differential Equations (North Ryde, 1986), Volume 14 of Proc. Centre Math. Anal. Austral. Nat. Univ., pp. 210-231. Austral. Nat. Univ., Canberra (1986)

69. Mei, T.: Notes on matrix valued paraproducts. Indiana Univ. Math. J. 55(2), 747-760 (2006)

70. Meyer, S., Wilke, M.: Optimal regularity and long-time behavior of solutions for the Westervelt equation. Appl. Math. Optim. 64(2), 257-271 (2011)

71. Meyries, M.: Global attractors in stronger norms for a class of parabolic systems with nonlinear boundary conditions. Nonlinear Anal. 75(5), 2922-2935 (2012)

72. Meyries, M., Schnaubelt, R.: Interpolation, embeddings and traces of anisotropic fractional Sobolev spaces with temporal weights. J. Funct. Anal. 262(3), 1200-1229 (2012)

73. Meyries, M., Schnaubelt, R.: Maximal regularity with temporal weights for parabolic problems with inhomogeneous boundary conditions. Math. Nachr. 285(8-9), 1032-1051 (2012)

74. van Neerven, J.M.A.M.: $\gamma$-radonifying operators-a survey. In: The AMSI-ANU Workshop on Spectral Theory and Harmonic Analysis, Volume 44 of Proc. Centre Math. Appl. Austral. Nat. Univ., pp. 1-61. Austral. Nat. Univ., Canberra (2010)

75. van Neerven, J.M.A.M., Veraar, M.C., Weis, L.W.: Maximal $L^{p}$-regularity for stochastic evolution equations. SIAM J. Math. Anal. 44(3), 1372-1414 (2012)

76. van Neerven, J.M.A.M., Veraar, M.C., Weis, L.W.: Stochastic maximal $L^{p}$-regularity. Ann. Probab. 40(2), 788-812 (2012)

77. van Neerven, J.M.A.M., Veraar, M.C., Weis, L.W.: On the $R$-boundedness of stochastic convolution operators. Positivity 19(2), 355-384 (2015)

78. Pazy, A.: Semigroups of Linear Operators and Applications to Partial Differential Equations, Volume 44 of Applied Mathematical Sciences. Springer, New York (1983)

79. Pierre, M.: Global existence in reaction-diffusion systems with control of mass: a survey. Milan J. Math. 78(2), 417-455 (2010)

80. Portal, P., Štrkalj, Ž.: Pseudodifferential operators on Bochner spaces and an application. Math. Z. 253(4), 805-819 (2006)

81. Prüss, J.: Maximal regularity for evolution equations in $L_{p}$-spaces. Conf. Semin. Mat. Univ. Bari 285, 1-39 (2003) (2002)

82. Prüss, J., Schnaubelt, R.: Solvability and maximal regularity of parabolic evolution equations with coefficients continuous in time. J. Math. Anal. Appl. 256(2), 405-430 (2001)

83. Prüss, J., Simonett, G.: Maximal regularity for evolution equations in weighted $L_{p}$-spaces. Arch. Math. 82(5), 415-431 (2004)

84. Prüss, J., Vergara, V., Zacher, R.: Well-posedness and long-time behaviour for the non-isothermal cahnHilliard equation with memory. Discrete Contin. Dyn. Syst. 26(2), 625-647 (2010)

85. Saal, J.: Wellposedness of the tornado-hurricane equations. Discrete Contin. Dyn. Syst. 26(2), 649-664 (2010)

86. Schnaubelt, R.: Asymptotic behaviour of parabolic nonautonomous evolution equations. In: Functional Analytic Methods for Evolution Equations, Volume 1855 of Lecture Notes in Math, pp. 401-472. Springer, Berlin (2004)

87. Stein, E.M.: Harmonic Analysis: Real-Variable Methods, Orthogonality, and Oscillatory Integrals, Volume 43 of Princeton Mathematical Series. Princeton University Press, Princeton (1993). With the assistance of Timothy S. Murphy, Monographs in Harmonic Analysis, III

88. Tanabe, H.: Equations of Evolution, Volume 6 of Monographs and Studies in Mathematics. Pitman (Advanced Publishing Program), Boston (1979)

89. Tanabe, H.: Functional Analytic Methods for Partial Differential Equations, Volume 204 of Monographs and Textbooks in Pure and Applied Mathematics. Marcel Dekker Inc., New York (1997)

90. Triebel, H.: Interpolation Theory, Function Spaces, Differential Operators, 2nd edn. Johann Ambrosius Barth, Heidelberg (1995) 
91. Weis, L.: A new approach to maximal $L_{p}$-regularity. In: Evolution Equations and their Applications in Physical and Life Sciences (Bad Herrenalb, 1998), Volume 215 of Lecture Notes in Pure and Appl. Math., pp. 195-214. Dekker, New York (2001)

92. Weis, L.: Operator-valued Fourier multiplier theorems and maximal $L_{p}$-regularity. Math Ann. 319(4), 735-758 (2001)

93. Weis, L.: The $H^{\infty}$ holomorphic functional calculus for sectorial operators-a survey. In: Partial Differential Equations and Functional Analysis, Volume 168 of Oper. Theory Adv. Appl., pp. 263-294. Basel, Birkhäuser (2006)

94. Yagi, A.: Abstract quasilinear evolution equations of parabolic type in Banach spaces. Boll. Un. Mat. Ital. B (7) 5(2), 341-368 (1991)

95. Yoshikawa, S., Pawlow, I., Zajączkowski, W.M.: Quasi-linear thermoelasticity system arising in shape memory materials. SIAM J. Math. Anal. 38(6), 1733-1759 (2007). (electronic) 